MATHEMATICS OF COMPUTATION

Volume 65, Number 216

October 1996, Pages 1403-1429

\title{
THE $p$ AND $h p$ VERSIONS OF THE FINITE ELEMENT METHOD FOR PROBLEMS WITH BOUNDARY LAYERS
}

\author{
CHRISTOPH SCHWAB AND MANIL SURI
}

Dedicated to Professor Ivo Babuška on the occasion of his seventieth birthday

\begin{abstract}
We study the uniform approximation of boundary layer functions $\exp (-x / d)$ for $x \in(0,1), d \in(0,1]$, by the $p$ and $h p$ versions of the finite element method. For the $p$ version (with fixed mesh), we prove super-exponential convergence in the range $p+1 / 2>e /(2 d)$. We also establish, for this version, an overall convergence rate of $\mathcal{O}\left(p^{-1} \sqrt{\ln p}\right)$ in the energy norm error which is uniform in $d$, and show that this rate is sharp (up to the $\sqrt{\ln p}$ term) when robust estimates uniform in $d \in(0,1]$ are considered. For the $p$ version with variable mesh (i.e., the $h p$ version), we show that exponential convergence, uniform in $d \in(0,1]$, is achieved by taking the first element at the boundary layer to be of size $\mathcal{O}(p d)$.

Numerical experiments for a model elliptic singular perturbation problem show good agreement with our convergence estimates, even when few degrees of freedom are used and when $d$ is as small as, e.g., 10 $10^{-8}$. They also illustrate the superiority of the $h p$ approach over other methods, including a low-order $h$ version with optimal "exponential" mesh refinement.

The estimates established in this paper are also applicable in the context of corresponding spectral element methods.
\end{abstract}

\section{INTRODUCTION}

Our goal in this paper is to develop the approximation theory for boundary layer functions

$$
u(x)=\exp (-a x / d), \quad 0<x<L,
$$

where $d \in(0,1]$ is a small parameter that can approach zero, $a>0$ is a constant and $L \geq 1$ is a typical length scale of the problem under consideration. We are interested in obtaining convergence estimates that are robust, i.e., uniform in $d$, when (1.1) is approximated by piecewise polynomials via $p$ and $h p$ type numerical schemes.

Boundary layers (1.1) arise as solution components in singularly perturbed elliptic boundary value problems, a model example of which is

$$
L_{d} u_{d}:=-d^{2} u_{d}^{\prime \prime}(x)+a^{2} u_{d}(x)=f(x), \quad x \in I=(-1,1),
$$

Received by the editor March 7, 1995.

1991 Mathematics Subject Classification. Primary 65N30, 35B30, 65N15.

Key words and phrases. Boundary layer, singularly perturbed problem, $p$ version, $h p$ version, spectral element method.

This work was supported in part by the Air Force Office of Scientific Research, Air Force Systems Command, USAF under Grant F49620-92-J-0100.

(C)1996 American Mathematical Society 


$$
u_{d}( \pm 1)=\alpha^{ \pm}
$$

A large body of literature has been devoted to their effective resolution. Most available references analyze the convergence of finite difference or finite element schemes of fixed (usually low) polynomial degree in conjunction with various mesh refinements (the $h$ version); see, e.g., $[4,6,13,18,19]$, and the references therein.

If the mesh refinement is quasi-uniform (or, more generally, independent of $d$ ), either on the whole domain or locally near the boundary, then the optimal (algebraic) decrease in the global error is observed provided a condition of the form $h \leq C d$ is met ( $h$ being the mesh spacing parameter). Such methods are nonrobust, in a sense made precise in $\S 3$. In practical terms, the amount of discretization required with such schemes for satisfactory resolution of the boundary layers may be infeasible when $d$ is very small. On the other hand, strongly graded $d$-dependent mesh refinement, like the one from [20] presented in $\S 6$, does lead to robust convergence, at an optimal rate that is algebraic (see $[4,16,18,19,20]$, where this and other graded meshes are discussed).

An alternative approach is to increase the polynomial degree and keep the mesh fixed, i.e., use a $p$ version or spectral element method. In [5], various such schemes (Galerkin, Tau and Collocation) have been considered for the special case of (1.2)(1.3) where $f \equiv 0, \alpha^{+}=1, \alpha^{-}=0$, using a Chebyshev-weighted spectral approximation. In this paper, we consider the unweighted Galerkin $p$ version/spectral element approximation. We provide a detailed study of the approximation theory for this method, showing that an asymptotic superexponential convergence rate for the error in the energy norm is achieved for $\tilde{p}:=p+1 / 2>e /(2 d)$. We also provide estimates for this error in the preasymptotic phase when $d$ is small, showing that (A) for $(3 /(4 d))^{1 / 2} \leq \tilde{p} \leq 2 / d$, the error is bounded by $C \exp \left(-\tilde{p}^{2} d / 3\right)$ and (B) for $\tilde{p} \leq K d^{-\frac{1}{2}}$, the error is bounded by $C p^{-1}$ (numerical experiments in $\S 6$ are in agreement with these rates). The results we prove for a single element also hold when a fixed mesh with several elements is used. Using our various estimates, we establish that for the pure $p$ version on fixed meshes, the overall robust rate, uniform in $d$, is $\mathcal{O}\left(p^{-1} \sqrt{\ln p}\right)$ and, up to the $\sqrt{\ln p}$ term, this is the best possible. Note that this rate is essentially double the uniform rate of $O\left(h^{\frac{1}{2}}\right)$ achievable (for the global error) by the $h$ version with quasi-uniform meshes (Theorem A.1(ii) of [13]). It is also double the uniform rate for $p$ version/spectral element methods that can be established from the results in [5]. (Since the methods in [5] involve a weighted projection, the estimates there are in (stronger) weighted norms.)

The $p$-type results in [5] can be considerably improved by using special "mapped" polynomials in the spectral element method. This is shown in $[8,7]$, where singular mappings of appropriately high order are used to establish algebraic rates of convergence that deteriorate relatively slowly as $d \rightarrow 0$. However, these estimates are still not uniform in $d$, and therefore, not robust in our sense.

Our main result in this paper shows that excellent robust rates for the uniform approximation of functions (1.1) can be achieved by using, instead of the pure $p /$ spectral version, a variable mesh with only one more element. More precisely, a robust exponential rate can be obtained by using the $p$ version on two elements, where the first one is of size $\mathcal{O}(p d)$. (For problems like (1.2)-(1.3), three elements are needed, owing to boundary layers at either end - see $\S 6$.) We call this an $h p$ version since the size (though not the number) of elements changes, as does $p$. (More appropriately, it is an " $r p$ " method.) Note that an exponential rate is not 
possible with either the $h$ version or the pure $p /$ spectral version - the estimates obtained in the papers above are all algebraic. Finite element computations for (1.2)-(1.3) presented in $\S 6$ confirm the theoretical convergence estimates obtained here and clearly show the dramatic superiority of this robust $h p$ FEM over other methods, especially for small $d$.

Although we concentrate here only on the approximation theory for the onedimensional function (1.1) applied to one-dimensional problems like (1.2), the scope of our results is wider. This is due to the fact that solutions to singularly perturbed problems over two-dimensional domains $\omega$, arising, e.g., in beam, plate and shell theory, as well as in reaction-diffusion and certain fluid dynamics problems, also exhibit boundary layers, which are of the form

$$
u_{B L}(s, x)=C(s) \exp (-a \rho(x) / d), \quad 0<s<T, \quad 0<\rho<\rho_{0} .
$$

Here $s, \rho$ denote, respectively, the arc length and normal distance to the boundary, of a point $x$ in a neighborhood of $\partial \omega$, and the function $C(s)$ is smooth. For several problems of practical interest, decompositions of the solution into a regular part and such boundary layers $u_{B L}(s, x)$ have been obtained in the literature; cf. [1] for the Reissner-Mindlin plate, [17] for beam theory, [11, 12] for shells. Similar decompositions arise also in three-dimensional problems (then, however, $s=\left(s_{1}, s_{2}\right)$ are coordinates in the boundary manifold). The key observation from (1.4) is that since $C(s)$ is smooth, the boundary layer phenomenon is essentially one-dimensional, namely, in the direction normal to $\partial \omega$. Hence, the crucial aspect of the FE approximation of such functions is how the $\mathrm{FE}$ spaces are designed in the $\rho$ direction, i.e., how the function (1.1) is approximated in one dimension. Using our results, therefore, we can construct two- and three-dimensional FE spaces (with robust exponential convergence) for the functions (1.4), e.g., using tensor product spaces in the $(s, \rho)$ coordinates. See $[15,16]$. Note that "brute force" mesh refinement will be even less competitive in two dimensions and practically impossible in three dimensions.

The outline of this paper is as follows. In $\S 2$ we present an asymptotic expansion for the solution of the model problem (1.2)-(1.3) which includes the boundary layers. The proof uses standard techniques and is provided for completeness in the Appendix. In $\S 3$, we describe the finite element methods and error measures to be analyzed. We also define the concept of robustness, using a definition from [2]. Section 4 is devoted to the convergence analysis of the $p$ version FEM. In $\S 5$, we consider an $h p$ version for which we prove a robust exponential convergence rate in various norms. Finally, in $\S 6$ we present numerical experiments comparing, in particular, the $p$ and $h p$ version FEMs analyzed here with an $h$ version from [20], based on asymptotically optimal meshes. We show that the $h p$ version consistently outperforms the other versions and that high accuracy can be achieved with few degrees of freedom for arbitrarily small $d$ (we take values of $d$ as small as $10^{-8}$ ).

Throughout, $H^{k}(I)$ will denote the Sobolev space of order $k \in \mathbb{N}_{0}$ on an interval $I \subset \mathbb{R}$, with $H^{0}(I)=L_{2}(I)$ and $\|\cdot\|_{k, I},|\cdot|_{k, I}$ denoting the norm and seminorm as usual. Whenever there is no confusion about the domain, we omit the subscript $I$. For $u, v \in L_{2}(I)$, we denote by $(u, v)$ the $L_{2}$ inner product. Also, $H_{0}^{1}(I)=\{u \in$ $\left.H^{1}(I): u( \pm 1)=0\right\}, H_{D}^{1}(I)=\left\{u \in H^{1}(I): u( \pm 1)=\alpha^{ \pm}\right\}$and $H^{-1}(I)=\left(H_{0}^{1}\right)^{*}$, the dual space. Throughout the paper, $C, K$ will denote generic constants, while $C_{i}, \tilde{C}_{i}$ will denote constants that are explicitly given or can be easily estimated from the exposition. 


\section{Regularity OF THE MODEL PROBLEM}

The variational formulation of the model problem (1.2)-(1.3) reads: Find $u_{d} \in$ $H_{D}^{1}(I)$ such that

$$
B_{d}\left(u_{d}, v\right)=(f, v) \quad \forall v \in H_{0}^{1}(I)
$$

Here, $f \in H^{-1}(I)$ and

$$
B_{d}(u, v):=\int_{I}\left\{d^{2} u^{\prime} v^{\prime}+a^{2} u v\right\} d x
$$

For every $f \in H^{-1}(I)$ the problem (2.1) admits a unique solution $u_{d} \in H_{D}^{1}(I)$, and if $f \in H^{k}(I)$, then $u_{d} \in H^{k+2}(I) \cap H_{D}^{1}(I)$. This regularity, however, is nonuniform in $d$ since in the a priori "shift" estimate

$$
\left\|u_{d}\right\|_{k+2} \leq C(k, d)\|f\|_{k}, \quad k=0,1,2, \ldots,
$$

the constant $C$ strongly depends on $d$. The following theorem, the proof of which can be found in the Appendix, presents a decomposition of $u_{d}$ into a smooth part $u_{d}^{M}(x)$ and boundary layers

$$
u_{a, d}(x)=\exp (-a(1+x) / d), \quad \bar{u}_{a, d}(x)=\exp (-a(1-x) / d) .
$$

Theorem 2.1. Let $f \in H^{4 M+2}(I)$ for some $M \in \mathbb{N}$. Then

$$
u_{d}(x)=u_{d}^{M}(x)+A_{d}^{M} u_{a, d}(x)+B_{d}^{M} \bar{u}_{a, d}(x),
$$

where $u_{d}^{M}(x)$ satisfies the following regularity estimate uniformly in $d$ for $\ell=$ $0,1, \ldots, 2 M$ :

$$
\left|u_{d}^{M}\right|_{\ell} \leq a^{-1}(d / a)^{2 M+2-\ell}|f|_{(2 M+2)}+2 a^{-2} \sum_{k=0}^{M+1}(d / a)^{2 k}|f|_{2 k+\ell}
$$

Further,

$$
\left|A_{d}^{M}\right|+\left|B_{d}^{M}\right| \leq C(a)\left\{\left|\alpha^{+}\right|+\left|\alpha^{-}\right|+\sum_{k=0}^{M}(d / a)^{2 k}\left(\left|f^{(2 k)}(-1)\right|+\left|f^{(2 k)}(+1)\right|\right)\right\},
$$

where $C(a)$ is independent of $M$ and $d$.

For any interval $\tilde{I}$ let $\Pi_{n}(\tilde{I})$ denote the set of polynomials on $\tilde{I}$ of degree $\leq n$. The following result follows by Remark 6.1 in the Appendix.

Corollary 2.1. Let $f \in \Pi_{2 M+1}(I)$; then $u_{d}^{M} \in \Pi_{2 M+1}(I)$ in (2.5).

Remark 2.1. Analogous results hold when the Dirichlet end conditions (1.3) are replaced by Neumann or mixed boundary conditions.

For $f$ smooth enough (i.e., $M$ large enough), we see from Theorem 2.1 that the regularity of $u_{d}$ (in terms of $d$ ) will be determined by the boundary layer terms. We have, in fact, by $(2.5)-(2.7)$,

$$
\left|u_{d}\right|_{\ell} \leq\left|u_{d}^{M}\right|_{\ell}+\left|A_{d}^{M}\right|\left|u_{a, d}\right|_{\ell}+\left|B_{d}^{M}\right|\left|\bar{u}_{a, d}\right|_{\ell} \leq C\left(1+\left|u_{a, d}\right|_{\ell}+\left|\bar{u}_{a, d}\right|_{\ell}\right),
$$

where the constant $C$ depends upon $a, f$ and $\alpha^{ \pm}$but is independent of $d$. For the function $u_{a, d}$, we have for $\ell=0,1,2, \ldots$

$$
\left|u_{a, d}\right|_{\ell}=\left(\frac{d}{a}\right)^{\frac{1}{2}-\ell}\left[\frac{1-e^{-4 a / d}}{2}\right]^{1 / 2} \approx C d^{\frac{1}{2}-\ell}
$$


so that (2.9) and its analog for $\left|\bar{u}_{a, d}\right|_{\ell}$, substituted in (2.8), gives an upper bound for $\left|u_{d}\right|_{\ell}$. Since, except for special cases, the coefficients $A_{d}^{M}, B_{d}^{N}$ are nonzero, we see that the following equivalence generally holds:

$$
\left|u_{d}\right|_{\ell} \approx C\left(1+d^{\frac{1}{2}-\ell}\right), \quad \ell=0,1, \ldots, 2 M .
$$

To conclude this section, we define the following solution spaces, which will be used later:

$$
\begin{aligned}
& H_{d, M}^{B}=\left\{u_{d} \mid u_{d} \text { is a solution of }(1.2)-(1.3) \text { with } f \in H^{4 M+2}(I),\right. \\
&\left.\|f\|_{4 M+2} \leq B,\left|\alpha^{ \pm}\right| \leq B\right\}, \\
& H_{d, \Pi_{n}}^{B}=\left\{u_{d} \mid u_{d} \text { is a solution of }(1.2)-(1.3) \text { with } f \in \Pi_{n}(I)\right. \text { such that } \\
&\text { all coefficients in } \left.f \text { are absolutely bounded by } B,\left|\alpha^{ \pm}\right| \leq B\right\} .
\end{aligned}
$$

\section{The FINITE ELEMENT METHOD}

For any finite-dimensional subspace $S$ of $H^{1}(I)$, denote $S_{D}=S \cap H_{D}^{1}(I), S_{0}=$ $S \cap H_{0}^{1}(I)$. Then a finite element approximation $u_{d}^{S}$ of $u_{d}$ is obtained by restricting both sides of the weak formulation (2.1) to finite-dimensional subspaces: Find $u_{d}^{S} \in S_{D}$ such that

$$
B_{d}\left(u_{d}^{S}, v\right)=(f, v) \quad \forall v \in S_{0} .
$$

For every $d \in(0,1]$ there exists a unique solution $u_{d}^{S} \in S_{D}$ of (3.1).

We will be interested in spaces $S$ of piecewise polynomials on $I$ characterized by the mesh-degree combination $\Sigma=(\Delta, \vec{p})$, defined as follows. Let the $m+1 \geq 2$ nodal points

$$
-1=: x_{0}<x_{1}<x_{2}<\cdots<x_{m-1}<x_{m}:=1
$$

be given; then the mesh $\Delta$ is defined by

$$
\Delta=\left\{I_{i}\right\}_{i=1}^{m}, \quad I_{i}=\left(x_{i-1}, x_{i}\right), \quad h_{i}=\left|I_{i}\right|=x_{i}-x_{i-1}
$$

(we will also write $\Delta=\left\{x_{0}, x_{1}, \ldots, x_{m}\right\}$ where convenient).

The degree vector $\vec{p}$ is defined by

$$
\vec{p}=\left(p_{1}, p_{2}, \ldots, p_{m}\right) .
$$

Then

$$
S(\Sigma)=\left\{u:\left.u\right|_{I_{i}} \in \Pi_{p_{i}}\left(I_{i}\right), \quad I_{i} \in \Delta\right\} \cap C^{0}[-1,1] .
$$

Obviously, $S(\Sigma) \subset H^{1}(I)$ and

$$
\operatorname{dim} S(\Sigma)=1+\sum_{i=1}^{m} p_{i}, \quad N=\operatorname{dim} S_{D}(\Sigma)=\operatorname{dim} S(\Sigma)-2 .
$$

By $(2.1),(3.1)$,

$$
B_{d}\left(u_{d}-u_{d}^{S}, v\right)=0 \quad \forall v \in S_{0}(\Sigma),
$$

so that $e_{d}^{S}=u_{d}-u_{d}^{S}$ satisfies

$$
\left\|e_{d}^{S}\right\|_{d}=\inf _{\chi \in S_{D}(\Sigma)}\left\|u_{d}-\chi\right\|_{d} .
$$

Here the energy norm $\|\cdot\|_{d}, 0<d \leq 1$, is defined by

$$
\|v\|_{d}=\left(B_{d}(v, v)\right)^{1 / 2}=\left(d^{2}|v|_{1}^{2}+\|v\|_{0}^{2}\right)^{1 / 2} \approx d|v|_{1}+\|v\|_{0} .
$$


The question we wish to explore here is the design of the spaces $S(\Sigma)$ such that $\left\|e_{d}^{S}\right\|_{d}$ has a good convergence rate $g(N) \rightarrow 0$ as $N \rightarrow \infty$ independent of $d$. To do this, we recall the definition of robustness from [2].

Definition 3.1. The FEM for problem (3.1) using spaces $S_{D}(\Sigma)$ is robust with uniform order $g(N)$ for $0<d \leq 1$ with respect to solution sets $\mathcal{H}_{d}=H_{d, M}^{B}$ (or $\left.H_{d, \Pi_{n}}^{B}\right)$ and error measures $E_{d}=\|\cdot\|_{d}$ if and only if

$$
\lim _{N \rightarrow \infty}\left(\left(\sup _{d \in(0,1]} \sup _{u_{d} \in \mathcal{H}_{d}} E_{d}\left(u_{d}-u_{d}^{N}\right)\right) \frac{1}{g(N)}\right)=C<\infty .
$$

Although we concentrate here primarily on the energy norm, other error measures could be considered as well: the $L_{2}$ norm obviously follows as a corollary, while the maximum norm is considered in Corollary 5.1. Note that by (2.10), the unscaled $H^{1}$ norm of $u_{d}$ is not bounded uniformly for $d \in(0,1]$, so that we cannot expect robustness with uniform order in this norm (see, e.g., estimates (4.3) in [13]).

Let $u_{d} \in H_{d, M}^{B}$. Using Theorem 2.1 and (3.8), we see immediately that for the energy norm,

$$
\begin{aligned}
& E_{d}\left(u_{d}-u_{d}^{N}\right)=\left\|e_{d}^{S}\right\|_{d} \leq \inf _{\chi \in S_{D}}\left\|\left(u_{d}^{M}+A_{d}^{M} u_{a, d}+B_{d}^{M} \bar{u}_{a, d}\right)-\chi\right\|_{d} \\
& \leq \inf _{\substack{\chi_{1} \in S \\
\chi_{1}( \pm 1)=u_{d}^{M}( \pm 1)}}\left\|u_{d}^{M}-\chi_{1}\right\|_{d} \\
& +\left|A_{d}^{M}\right| \inf _{\substack{\chi_{2} \in S \\
\chi_{2}( \pm 1)=u_{a, d}( \pm 1)}}\left\|u_{a, d}-\chi_{2}\right\|_{d}+\left|B_{d}^{M}\right| \inf _{\substack{\chi_{3} \in S \\
\chi_{3}( \pm 1)=\bar{u}_{a, d}}}\left\|\bar{u}_{a, d}-\chi_{3}\right\|_{d} .
\end{aligned}
$$

Assume the space $S(\Sigma)$ has the following approximation property:

$$
\inf _{\substack{\chi \in S \\ \chi( \pm 1)=u( \pm 1)}}\|u-\chi\|_{1} \leq F(N, k)\|u\|_{k}, \quad k=1,2, \ldots,
$$

where $F(N, k)$ is some (optimal) approximation order (i.e., $F(N, k) \rightarrow 0$ as $N \rightarrow$ $\infty)$. Then for $u_{d} \in H_{d, M}^{B}$, by Theorem 2.1, the first infimum in (3.10) will tend to zero at the rate $K F(N, 2 M)$ as $N \rightarrow \infty$, where $K$ is a constant independent of $d$ ( $K$ only depends upon $B$ and $M$ ). Also, we may assume by Theorem 2.1 that $\left|A_{d}^{M}\right|,\left|B_{d}^{M}\right| \leq K$, so that the second infimum in (3.10) will decrease at the rate $K \sqrt{a} \Phi\left(\frac{d}{a}, S\right)$, where

$$
\begin{aligned}
\Phi(d, S)= & \inf _{\substack{\chi \in S \\
\chi( \pm 1)=u_{1, d}( \pm 1)}}\left\|u_{1, d}-\chi\right\|_{d} \\
= & \inf _{\chi \in S}\left\{d^{2}\left|u_{1, d}-\chi\right|_{1}^{2}+\left\|u_{1, d}-\chi\right\|_{0}^{2}\right\}^{1 / 2} .
\end{aligned}
$$

By symmetry about $x=0$, the last term in (3.10) will also have the same bound. Then it may be shown that our FEM will be robust in the sense of Definition 3.1 if and only if $\Phi(d, S)$ in (3.12) can be bounded independently of $d$, i.e.,

$$
\sup _{d \in(0,1]} \Phi(d, S) \leq G(N) .
$$

In that case, by (3.10)-(3.13) and Definition 3.1, our FEM will be robust with uniform order

$$
g(N)=C \max \{F(N, 2 M), G(N)\} .
$$


We will use the following related definition.

Definition 3.2. The spaces $S(\Sigma)$ will be said to approximate boundary layers $u_{1, d}$ robustly at the rate $G(N)$ in the energy norm if and only if (3.13) holds.

Remark 3.1. Our main concern in (3.14) is the rate $G(N)$, i.e., finding spaces $S(\Sigma)$ such that (3.13) holds with $G(N) \rightarrow 0$ uniformly at a sufficiently fast rate. This is because in general, $G(N)$ will be the dominant term in (3.14), the idea being that $M$ is large enough so that $F(N, 2 M)$ is sufficiently small. For the $h p$ spaces in $\S 5$, however, $G(N) \rightarrow 0$ exponentially, so that the algebraic rate $F(N, 2 M)$ achieved by assuming regularity in terms of finite $M$ will dominate as $N$ becomes sufficiently large. This technical problem could be overcome by restricting the set of solutions $\mathcal{H}_{d}$ in Definition 3.1 to those for which the first infimum in (3.10) decays exponentially (or sufficiently fast). In particular, choosing $\mathcal{H}_{d}=H_{d, \Pi_{n}}^{B}$ will make this infimum vanish for suitable $S(\Sigma)$ (see Theorem 5.2 ahead).

Remark 3.2. The FE spaces satisfying (3.13) constructed in this paper and the estimates $G(N)$ established for them are also applicable to various other problems where the solution can be decomposed into boundary layers and smooth terms.

\section{Approximation Results For the $p$ Version}

In this section, we will prove asymptotic error estimates for $\Phi(d, S)$ given by (3.12) as $p \rightarrow \infty$, in the case that a single element $I=(-1,1)$ is used, i.e., $S(\Sigma)=$ $\Pi_{p}(I)$. Our first estimate (4.1) will be valid uniformly in $d$ for the range $\tilde{p}>e / 2 d$. (For any integer $k$, we write $\tilde{k}=k+\frac{1}{2}$.) We will also provide separate estimates (again uniform in $d$ ) for the preasymptotic ranges $\sqrt{3 / 4 d} \leq \tilde{p} \leq 2 / d$ and $1<\tilde{p}<$ $K d^{-\frac{1}{2}}$. Our final theorem will establish a uniform robustness rate of $C p^{-1} \sqrt{\ln p}$ for the $p$ version over a fixed mesh, which will be shown to be optimal (up to the factor $\sqrt{\ln p}$ ).

In order to estimate $\Phi(d, S)$, we will use the following lemma from [3, Chapter $3]$, that will give a concurrent approximation of $u_{1, d}(x)$ in the $L_{2}(I)$ norm and $H^{1}(I)$ seminorm.

Lemma 4.1. Let $u, u^{\prime} \in L_{2}(I)$ and denote by

$$
a_{n}=\tilde{n} \int_{-1}^{1} u^{\prime}(x) P_{n}(x) d x
$$

the Legendre coefficients of $u^{\prime}(x)$. Then there exists $\chi \in \Pi_{p}(I)$ such that

$$
\begin{gathered}
\chi( \pm 1)=u( \pm 1), \\
\left\|u^{\prime}-\chi^{\prime}\right\|_{0, I}^{2}=\sum_{n=p}^{\infty} \frac{\left|a_{n}\right|^{2}}{\tilde{n}}, \\
\|u-\chi\|_{0, I}^{2} \leq \sum_{n=p}^{\infty} \frac{\left|a_{n}\right|^{2}}{n(n+1) \tilde{n}}, \\
\left\|u^{\prime}-\chi^{\prime}\right\|_{0, I} \leq\left\|u^{\prime}-\xi^{\prime}\right\|_{0, I}
\end{gathered}
$$

for any $\xi \in \Pi_{p}(I)$ satisfying $\xi( \pm 1)=u( \pm 1)$.

For a proof, we refer to [3, Theorem 3.3.4]. 
Remark 4.1. The polynomial $\chi$ above is obtained as an antiderivative of the truncated Legendre expansion of $u^{\prime}$, of degree $p-1$. While by (4.5), this is optimal in the $H^{1}(I)$ seminorm, it is nonoptimal in the $\|\cdot\|_{d}$ norm. Nevertheless, Lemma 4.1 will be sufficient for our purposes here.

The estimates in (4.3), (4.4) obviously depend on the size of the Legendre coefficients $a_{n}$ in dependence on $d$ and $n$. The following lemma gives precise bounds for these coefficients for our function $u \equiv u_{1, d}$.

Lemma 4.2. Let $u \equiv u_{1, d}$ and $a_{n}$ be defined by (4.1). Then with $\tilde{n}=n+\frac{1}{2}$,

$$
\left(1-\frac{2 \nu_{0}}{\tilde{n}}\right) \leq \frac{a_{n}(d)}{\phi(n, d)} \leq\left(1-\frac{2 \nu_{0}}{\tilde{n}}\right)^{-1} \text { for } n=1,2, \ldots,
$$

where

$$
\begin{gathered}
\phi(n, d)=(-1)^{n+1} \frac{d^{-\frac{1}{2}} \tilde{n}^{\frac{1}{2}}}{\left(1+z^{2}\right)^{\frac{1}{4}}} e^{-\tilde{n}(z-\xi(z))}, \quad z=(\tilde{n} d)^{-1} \\
\xi(z)=\left(1+z^{2}\right)^{\frac{1}{2}}+\ln \left(\frac{z}{1+\left(1+z^{2}\right)^{1 / 2}}\right) \\
\nu_{0}=\frac{1}{6 \sqrt{5}}+\frac{1}{12} \approx 0.158 .
\end{gathered}
$$

Proof. Using (4.1) and the fact that $u_{1, d}^{\prime}=-d^{-1} u_{1, d}$, we have

$$
\begin{aligned}
a_{n} & =-\tilde{n} d^{-1} e^{-1 / d} \int_{-1}^{1} e^{-x / d} \frac{1}{2^{n} n !} \frac{d^{n}}{d x^{n}}\left(\left(x^{2}-1\right)^{n}\right) d x \\
& =(-1)^{n+1} d^{-n-1} e^{-1 / d} \frac{\tilde{n}}{2^{n} n !} \int_{-1}^{1}\left(1-x^{2}\right)^{n} e^{-x / d} d x .
\end{aligned}
$$

Hence, by formula 3.387 of [10],

$$
\begin{aligned}
a_{n}(d) & =(-1)^{n+1} d^{-n-1} e^{-1 / d} \frac{\tilde{n}}{2^{n}} \sqrt{\pi}(2 d)^{\tilde{n}} I_{\tilde{n}}\left(d^{-1}\right) \\
& =(-1)^{n+1} d^{-1 / 2} \sqrt{2 \pi} \tilde{n} e^{-1 / d} I_{\tilde{n}}\left(d^{-1}\right),
\end{aligned}
$$

where $I_{\tilde{n}}\left(d^{-1}\right)$ is the modified Bessel function ([10, 8.406]). Thus, to obtain the asymptotic behavior of $a_{n}(d)$, we must investigate $I_{\tilde{n}}\left(d^{-1}\right)$. To this end, we use asymptotic expansions of $I_{\nu}(\nu z)$ that are uniform for $z>0$. Such uniform expansions have been obtained by F.W.J. Olver (see [9] and the references therein).

Let $\nu=\tilde{n}=n+\frac{1}{2}$ and $z=(\nu d)^{-1}$; then

$$
e^{-1 / d} I_{\tilde{n}}\left(d^{-1}\right)=e^{-\nu z} I_{\nu}(\nu z) .
$$

It is shown in [9] that

$$
e^{-\nu z} I_{\nu}(\nu z)=\left(\frac{t}{2 \pi \nu}\right)^{\frac{1}{2}} e^{-\nu(z-\xi(z))} \frac{\sum_{s=0}^{m} \frac{\mathcal{U}_{s}(t)}{\nu^{s}}+\epsilon_{m}(\nu, t)}{1+\epsilon_{m}(\nu, 0)},
$$

where $t=\left(1+z^{2}\right)^{-1 / 2}, m \geq 0$ is an integer, and the $\mathcal{U}_{s}(t)$ are certain polynomials of degree $3 s$ in $t$ (see [9]), the first two of which are given by

$$
\mathcal{U}_{0}(t)=1, \quad \mathcal{U}_{1}(t)=\left(3 t-5 t^{3}\right) / 24 .
$$


The $\epsilon_{m}$ in (4.10) are estimated by ([9])

$$
\left|\epsilon_{m}(\nu, t)\right| \leq \frac{\nu}{\left(\nu-\nu_{0}\right)} \frac{\mathcal{V}_{t}^{1}\left(\mathcal{U}_{m+1}\right)}{\nu^{m+1}},
$$

where

$$
\mathcal{V}_{a}^{b}(\mathcal{U})=\int_{a}^{b}\left|\mathcal{U}^{\prime}(t)\right| d t \text { and } \nu_{0}=\mathcal{V}_{0}^{1}\left(\mathcal{U}_{1}\right)=\frac{1}{6 \sqrt{5}}+\frac{1}{12} .
$$

Simplifying (4.9) and using (4.10)-(4.13) with $m=0$ yields, with $\phi(n, d)$ as in (4.7), that

$$
a_{n}=\phi(n, d) \frac{1+\epsilon_{0}(\tilde{n}, t)}{1+\epsilon_{0}(\tilde{n}, 0)} .
$$

The assertion then follows since

$$
0<\frac{1+\epsilon_{0}(\tilde{n}, t)}{1+\epsilon_{0}(\tilde{n}, 0)} \leq\left(1-\frac{2 \nu_{0}}{\tilde{n}}\right)^{-1}
$$

Remark 4.2. The bounds (4.6) are quite sharp, since, for example, for $n \geq 1$ we obtain that

$$
0.7895 \leq 1-\frac{2 \nu_{0}}{\tilde{n}}, \quad\left(1-\frac{2 \nu_{0}}{\tilde{n}}\right)^{-1} \leq 1.2667 .
$$

Lemma 4.2 reduces the description of the asymptotic behavior of $a_{n}(d)$ to a discussion of the function $\phi(n, d)$. We then obtain the following bounds on the approximation errors (4.3), (4.4).

Lemma 4.3. We have

$$
\begin{gathered}
\left\|u^{\prime}-\chi^{\prime}\right\|_{0}^{2} \leq \sum_{n=p}^{\infty} \theta^{+}(n, d) e^{-2 \tilde{n}(z-\xi(z))}, \\
\|u-\chi\|_{0}^{2} \leq \sum_{n=p}^{\infty} \frac{1}{n(n+1)} \theta^{+}(n, d) e^{-2 \tilde{n}(z-\xi(z))}, \\
\left\|u^{\prime}-\chi^{\prime}\right\|_{0}^{2} \geq \sum_{n=p}^{\infty} \theta^{-}(n, d) e^{-2 \tilde{n}(z-\xi(z))},
\end{gathered}
$$

where $z=(\tilde{n} d)^{-1}, \xi(z)$ is as in (4.8) and

$$
\theta^{ \pm}(n, d):=\left(1-\frac{2 \nu_{0}}{\tilde{n}}\right)^{\mp 2}\left(d^{2}+\tilde{n}^{-2}\right)^{-\frac{1}{2}} .
$$

As is readily apparent from the expression for $\phi(n, d)$ in $(4.7)$, we can expect exponential decay of $a_{n}$ as $n \rightarrow \infty$ provided the function $z-\xi(z)$ is positive and of reasonable size. The following lemma provides bounds for $z-\xi(z)$ in terms of the asymptotes shown in Figure 1. The proof follows by elementary arguments.

Lemma 4.4. For any $z>0$, we have $z-\xi(z) \geq 0$. Moreover, the following bounds hold:

$$
\begin{gathered}
-(1+\ln (z / 2)) \leq z-\xi(z) \leq z-(1+\ln (z / 2)), \\
\frac{1}{2 z}-\frac{1}{24 z^{3}} \leq z-\xi(z) \leq \frac{1}{2 z} .
\end{gathered}
$$




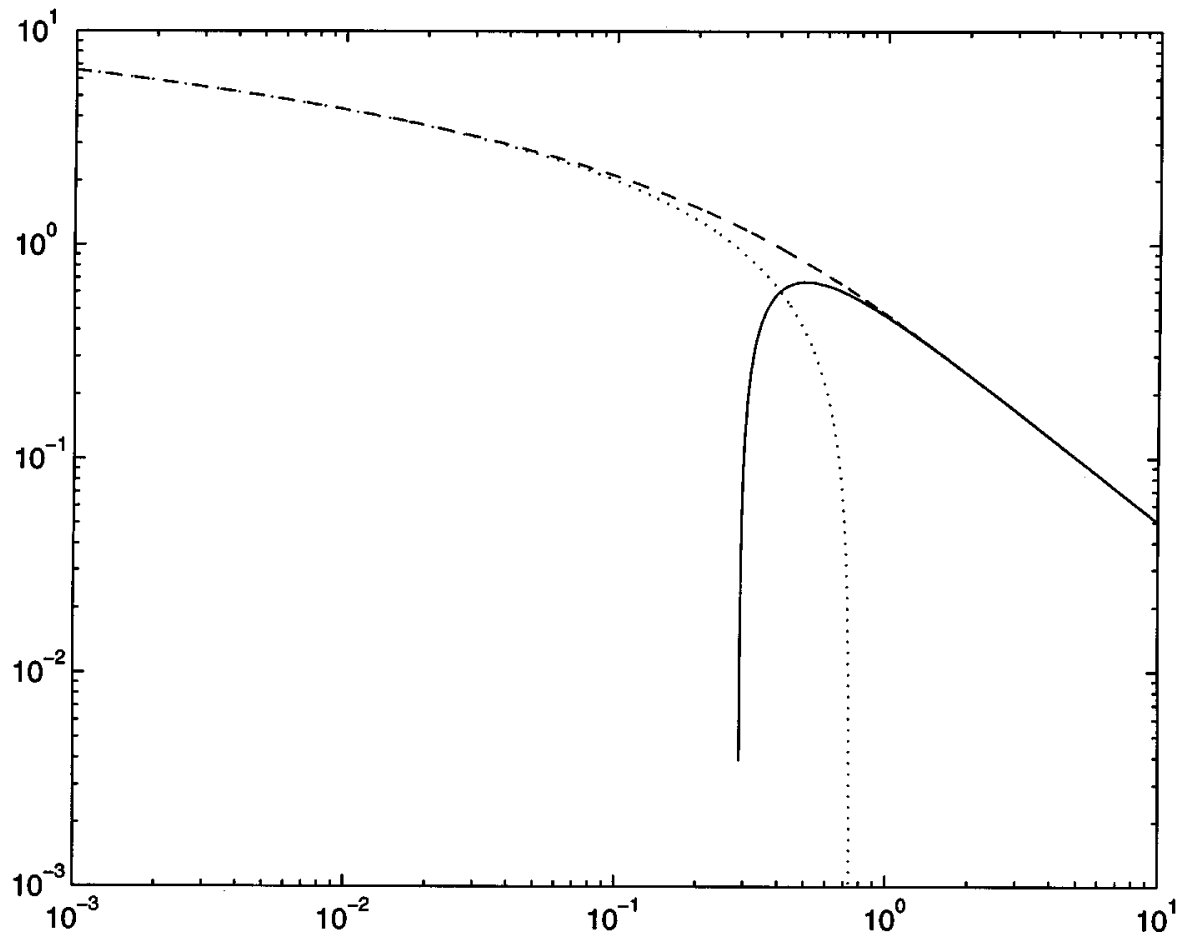

Figure 1. The function $z-\xi(z)(--)$ and its asymptotes $1 /(2 z)-$ $1 /\left(24 z^{3}\right)(-)$ and $-(1+\ln (z / 2))(\cdots)$

We prove now an error bound for sufficiently large $p(\tilde{p}>e / 2 d)$.

Theorem 4.1. Let $r:=e /(2 \tilde{p} d)<1$. Then for $u \equiv u_{1, d}$ there exists a polynomial $\chi \in \Pi_{p}(I)$ such that $\chi( \pm 1)=u( \pm 1)$ and

$$
\begin{gathered}
\left\|u^{\prime}-\chi^{\prime}\right\|_{0} \leq C_{1} d^{-1 / 2} r^{\tilde{p}}\left(1-r^{2}\right)^{-1 / 2} \\
\|u-\chi\|_{0} \leq C_{0} d^{1 / 2} r^{\tilde{p}}\left(1-r^{2}\right)^{-1 / 2} .
\end{gathered}
$$

Here, $C_{i}$ are independent of $p$ and $d$ (numerical values can be read off the proof).

Proof. By (4.14), (4.17), we must estimate the sum

$$
\begin{aligned}
S^{+} & =\sum_{n=p}^{\infty}\left(1-\frac{2 \nu_{0}}{\tilde{n}}\right)^{-2}\left(d^{2}+\tilde{n}^{-2}\right)^{-\frac{1}{2}} e^{-2 \tilde{n}(z-\xi(z))} \\
& \leq\left(1-\frac{4 \nu_{0}}{3}\right)^{-2} \sum_{n=p}^{\infty} d^{-1} e^{-2 \tilde{n}(z-\xi(z))}
\end{aligned}
$$

Using the lower bound in (4.18), we get

$$
\begin{aligned}
S^{+} & \leq\left(1-4 \nu_{0} / 3\right)^{-2} d^{-1} \sum_{n=p}^{\infty}\left(\frac{e}{2 \tilde{n} d}\right)^{2 \tilde{n}} \\
& =C_{1}^{2} d^{-1} \sum_{n=p}^{\infty} r^{2 \tilde{n}}=C_{1}^{2} d^{-1} r^{2 \tilde{p}}\left(1-r^{2}\right)^{-1} .
\end{aligned}
$$


This is (4.20). To prove (4.21), we observe that $r<1$ implies that

$$
\frac{1}{p(p+1)} \leq \frac{9}{8 \tilde{p}^{2}} \leq \frac{9}{8}\left(\frac{4 d^{2}}{e^{2}}\right)=\frac{9 d^{2}}{2 e^{2}}
$$

Hence,

$$
\frac{1}{p(p+1)} S^{+} \leq C_{0}^{2} d r^{2 \tilde{p}}\left(1-r^{2}\right)^{-1}, C_{0}^{2}=\frac{9}{2 e^{2}} C_{1}^{2},
$$

and (4.21) follows.

Corollary 4.1. Let $\Phi(d, S)$ be as in (3.12). Then for $r=e /(2 \tilde{p} d)<1$,

$$
\Phi(d, S) \leq C_{2} d^{1 / 2} r^{\tilde{p}}\left(1-r^{2}\right)^{-1 / 2}, \quad C_{2}=\left(C_{0}^{2}+C_{1}^{2}\right)^{1 / 2} .
$$

Remark 4.3. The asymptotic rate of convergence with respect to $p$ in (4.20) and hence (4.24) is optimal up to a constant depending on $d$, since by (4.16),

$$
\left\|u^{\prime}-\chi^{\prime}\right\|_{0}^{2} \geq \sum_{n=p}^{\infty} \theta^{-}(n, d) e^{-2 \tilde{n}(z-\xi(z))}=: S^{-} .
$$

Using the upper bound in (4.18) yields

$$
\begin{aligned}
S^{-} & \geq\left(1-\frac{2 \nu_{0}}{\tilde{p}}\right)^{2} \sum_{n=p}^{\infty}\left(d^{2}+\tilde{n}^{-2}\right)^{-\frac{1}{2}} e^{-2 / d} r^{2 \tilde{n}} \\
& \geq\left(1-\frac{4 \nu_{0}}{3}\right)^{2} e^{-2 / d} d^{-1} \sum_{n=p}^{\infty}\left(1+z^{2}\right)^{-1 / 2} r^{2 \tilde{n}} \\
& \geq\left(1-\frac{4 \nu_{0}}{3}\right)^{2}\left(1+4 / e^{2}\right)^{-\frac{1}{2}} e^{-2 / d} d^{-1} r^{2 \tilde{p}}\left(1-r^{2}\right)^{-1},
\end{aligned}
$$

since $z=(\tilde{n} d)^{-1} \leq(\tilde{p} d)^{-1}<2 / e$. Hence,

$$
\left\|u^{\prime}-\chi^{\prime}\right\|_{0} \geq C_{3} e^{-1 / d} d^{-1 / 2} r^{\tilde{p}}\left(1-r^{2}\right)^{-1 / 2} .
$$

Now $\chi$ is the same polynomial as in Lemma 4.1, so that (4.5) holds. Comparing the above estimate with (4.20), we see that (4.20), (4.24) are optimal in $p$ for any fixed $d>0$ as $p \rightarrow \infty$.

The estimates in Theorem 4.1 are useful for the case that $\tilde{p}$ is large compared to $e /(2 d)$. Such a situation arises in the next section, where this theorem will be applied. In actual practice, if $d$ is small, then it can be difficult to ever be in this asymptotic range of $\tilde{p}$. The computational results in $\S 6$ show that convergence is observed in the preasymptotic range $\tilde{p} \leq e /(2 d)$ as well. Therefore, we now obtain estimates for the rate of decrease of the error in the range $\sqrt{3 /(4 d)} \leq \tilde{p} \leq e /(2 d)$.

In Theorem 4.1, we used the bounds (4.18) as well as (4.19), the latter being sharper for the range $\tilde{p} \leq e /(2 d)$, i.e., $z \geq 2 / e$ (see Figure 1 ). It is seen that the two lower bounds for $z-\xi(z)$ in Lemma 4.4 intersect at the root of

$$
\frac{1}{2 z}-\frac{1}{24 z^{3}}+1+\ln \left(\frac{z}{2}\right)=0
$$

i.e., at $z^{*}=0.51388 \ldots$, which is close to 0.5 . Our estimate will therefore be valid for $z \geq 0.5$, i.e., in the extended range $\sqrt{3 /(4 d)} \leq \tilde{p} \leq 2 / d$. 
Theorem 4.2. Assume that $\sqrt{3 /(4 d)} \leq \tilde{p} \leq 2 / d$. Then, for $u \equiv u_{1, d}$, there exists a polynomial $\chi \in \Pi_{p}(I)$ such that $\chi( \pm 1)=u( \pm 1)$ and

$$
\begin{gathered}
\left\|u^{\prime}-\chi^{\prime}\right\|_{0}^{2} \leq C_{1}^{2}\left(\tilde{p}+\frac{3}{4 d}\right) \exp \left(-2 \tilde{p}^{2} d / 3\right)+C_{3} d^{-1}(e / 4)^{4 / d}, \\
\|u-\chi\|_{0}^{2} \leq \frac{9}{8} C_{1}^{2} \tilde{p}^{-2}\left(\tilde{p}+\frac{3}{4 d}\right) \exp \left(-2 \tilde{p}^{2} d / 3\right)+\frac{4}{15} C_{3} d(e / 4)^{4 / d} .
\end{gathered}
$$

Here the constants $C_{i}$ are independent of $p$ and $d$ (and are given in the proof below).

Proof. Let us define the index sets

$$
I_{1}(d)=\{n \in \mathbb{N}: \tilde{p} \leq \tilde{n}<2 / d\}, \quad I_{2}(d)=\{n \in \mathbb{N}: \tilde{n} \geq 2 / d\} .
$$

Then, taking $\chi$ to be the polynomial in Lemma 4.1, we have by (4.14)

$$
\left\|u^{\prime}-\chi^{\prime}\right\|_{0}^{2} \leq S_{1}+S_{2}, \quad S_{i}=\sum_{n \in I_{i}} \theta^{+}(n, d) e^{-2 \tilde{n}(z-\xi(z))} .
$$

The quantities $S_{1}$ and $S_{2}$ will be estimated using the lower bounds in (4.19) and (4.18), respectively. First, by (4.19),

$$
S_{1} \leq \sum_{n \in I_{1}(d)} \theta^{+}(n, d) \exp \left(-2 \tilde{n}\left(\frac{1}{2 z}-\frac{1}{24 z^{3}}\right)\right),
$$

where, since $n \in I_{1}(d)$, we have $0<z^{-1}=\tilde{n} d<2$, so that $0<z^{-3}<4 z^{-1}$. Hence,

$$
\frac{1}{2 z}-\frac{1}{24 z^{3}} \geq \frac{1}{2 z}-\frac{1}{6 z}=\frac{1}{3 z},
$$

and (4.27), (4.17) give (with $C_{1}=\left(1-4 \nu_{0} / 3\right)^{-1}$ as in (4.23))

$$
S_{1} \leq C_{1} \sum_{n \in I_{1}(d)} \tilde{n}\left(1+\tilde{n}^{2} d^{2}\right)^{-1 / 2} e^{-\frac{2}{3} \tilde{n}^{2} d} .
$$

Now the function $x e^{-\frac{2}{3} x^{2} d}$ attains its global maximum at $x=\sqrt{3 /(4 d)}$ and is decreasing for $x>\sqrt{3 /(4 d)}$. Hence,

$$
\begin{aligned}
S_{1} & \leq C_{1} \sum_{n \in I_{1}(d)} \tilde{n} e^{-\frac{2}{3} \tilde{n}^{2} d} \leq C_{1}\left(\tilde{p} e^{-\frac{2}{3} \tilde{p}^{2} d}+\int_{\tilde{p}}^{2 / d} x e^{-\frac{2}{3} x^{2} d} d x\right) \\
& \leq C_{1}\left(\tilde{p}+\frac{3}{4 d}\right) e^{-\frac{2}{3} \tilde{p}^{2} d} .
\end{aligned}
$$

For the term $S_{2}$, we use (4.18). Noting that $\tilde{n}^{-1} \leq d / 2$, we have

$$
S_{2} \leq \sum_{\tilde{n} \geq 2 / d} \theta^{+}(n, d)\left(\frac{e}{2 \tilde{n} d}\right)^{2 \tilde{n}} \leq \tilde{C}_{3} d^{-1} \sum_{\tilde{n} \geq 2 / d}\left(\frac{e}{4}\right)^{2 \tilde{n}},
$$

where $\tilde{C}_{3}=\left(1-\nu_{0}\right)^{-2}\left(\frac{4}{5}\right)^{1 / 2}$. Summing the geometric series leads to the last term in (4.25), where $C_{3}=\tilde{C}_{3}\left(1-e^{2} / 16\right)^{-1}$.

For the $L_{2}$ estimates, (4.15) gives

$$
\|u-\chi\|_{0}^{2} \leq \tilde{S}_{1}+\tilde{S}_{2}, \quad \tilde{S}_{i}=\sum_{n \in I_{i}(d)} \frac{1}{n(n+1)} \theta^{+}(n, d) e^{-2 \tilde{n}(z-\xi(z))}, \quad i=1,2 .
$$


It is easy to see that

$$
\tilde{S}_{1} \leq \frac{1}{p(p+1)} S_{1} \leq \frac{9}{8 \tilde{p}^{2}} S_{1}
$$

Also, for $n \in I_{2}(d)$, we have $n(n+1) \geq \frac{16-d^{2}}{4 d^{2}} \geq \frac{15}{4 d^{2}}$ for $d \leq 1$, so that

$$
\tilde{S}_{2} \leq \frac{4 d^{2}}{15} S_{2}
$$

The theorem follows.

Theorem 4.2 leads to the following corollary.

Corollary 4.2. For $\sqrt{3 /(4 d)} \leq \tilde{p} \leq 2 / d$,

$$
\Phi(d, S) \leq C_{4} e^{-\frac{\tilde{p}^{2} d}{3}}+C_{5}\left(\frac{e}{4}\right)^{\frac{2}{d}},
$$

where the constants $C_{i}$ are independent of $p, d$.

Proof. Using the definition of $\Phi(d, S)$, we obtain from Theorem 4.2,

$$
(\Phi(d, S))^{2} \leq C_{1}^{2}\left(\tilde{p}+\frac{3}{4 d}\right)\left(d^{2}+\frac{9}{8} \tilde{p}^{-2}\right) e^{-\frac{2}{3} \tilde{p}^{2} d}+\frac{19}{15} C_{3}\left(\frac{e}{4}\right)^{\frac{4}{d}} .
$$

Hence, (4.28) holds with $C_{5}^{2}=\frac{19}{15} C_{3}$ and

$$
C_{1}^{2}\left(\tilde{p} d^{2}+\frac{3}{4} d+\frac{9}{8} \tilde{p}^{-1}+\frac{27}{32} \frac{\tilde{p}^{-2}}{d}\right) \leq C_{1}^{2}\left(4 \tilde{p}^{-1}+\frac{3}{2} \tilde{p}^{-1}+\frac{9}{8} \tilde{p}^{-1}+\frac{9}{8}\right) \leq C_{4}^{2} .
$$

We see from Corollary 4.2 that for small $d$, since the term $C_{5}(e / 4)^{2 / d}$ is negligible, the first term $C_{4} \exp \left(-\left(\tilde{p}^{2} d\right) / 3\right)$ in $(4.28)$ will dominate. Hence, the error will decrease at an exponential rate in this range when $\tilde{p}^{2} d / 3$ is large enough. For $\tilde{p}>e /(2 d)$, a better estimate may be provided by Theorem 4.1. When $\tilde{p}^{2} d / 3$ is small (i.e., $\tilde{p}=K d^{-\frac{1}{2}}$ ), the estimate (4.28) deteriorates. We will therefore establish another bound, which is valid in the range $1<\tilde{p} \leq K d^{-\frac{1}{2}}$. First, we prove the following lemma.

Lemma 4.5. There exists a unique polynomial $\chi \in \Pi_{p}(I)$ that minimizes $\|\chi\|_{0}$ subject to the constraints $\chi( \pm 1)=\alpha^{ \pm}$. This polynomial $\chi$ satisfies

$$
\begin{gathered}
\frac{1}{C} \frac{\max \left(\left|\alpha^{+}\right|,\left|\alpha^{-}\right|\right)}{p} \leq\|\chi\|_{0} \leq C \frac{\max \left(\left|\alpha^{+}\right|,\left|\alpha^{-}\right|\right)}{p}, \\
\|\chi\|_{1} \leq C \max \left(\left|\alpha^{+}\right|,\left|\alpha^{-}\right|\right) p
\end{gathered}
$$

with $C>1$ a constant independent of $\alpha^{ \pm}, p$.

Proof. We may write $\chi$ in the Legendre series expansion satisfying the end constraints,

$$
\chi(x)=\sum_{k=0}^{p} a_{k} P_{k}(x), \quad \sum_{k=0}^{p} a_{k}( \pm 1)^{k}=\alpha^{ \pm} .
$$


Introducing Lagrange multipliers for the constraints, we get the minimization problem

$$
\min _{\vec{a}, \lambda_{+}, \lambda_{-}} F\left(\vec{a}, \lambda_{+}, \lambda_{-}\right)=\sum_{k=0}^{p} \omega_{k} a_{k}^{2}+\lambda_{+}\left(\sum_{k=0}^{p} a_{k}-\alpha^{+}\right)+\lambda_{-}\left(\sum_{k=0}^{p} a_{k}(-1)^{k}-\alpha^{-}\right),
$$

where $\omega_{k}=2 /(2 k+1)$. Let $A=p(p+1)^{2}(p+2) / 4$. Then it may be shown that the unique minimizer for the above is given by

$$
\begin{aligned}
& \lambda_{ \pm}=\frac{2}{A}\left(\alpha^{\mp}(-1)^{p} \frac{(p+1)}{2}-\alpha^{ \pm} \frac{(p+1)^{2}}{2}\right), \\
& a_{k}=\left(\alpha^{+}+(-1)^{k} \alpha^{-}\right) \frac{\left((p+1)^{2}-(-1)^{p+k}(p+1)\right)}{2 A \omega_{k}},
\end{aligned}
$$

from which the bounds for $\|\chi\|_{0}$ in (4.29) follow easily. The bound for $\|\chi\|_{1}$ follows by the inverse inequality for polynomials,

$$
|\chi|_{1} \leq C p^{-2}\|\chi\|_{0}
$$

Theorem 4.3. Assume that $1<\tilde{p} \leq K d^{-\frac{1}{2}}$ for some $K$ (which may depend upon $\tilde{p}, d)$. Then for such $p, d$,

$$
\Phi(d, S) \leq C K p^{-1}
$$

where the constant $C$ is independent of $K, p$ and $d$.

Proof. We note that for any $\chi \in \Pi_{p}(I)$ satisfying $\chi( \pm 1)=u_{1, d}( \pm 1)$,

$$
\Phi(d, S) \leq C\left(\left\|u_{1, d}\right\|_{d}+d|\chi|_{1}+\|\chi\|_{0}\right) .
$$

We use (2.9) to bound $\left\|u_{1, d}\right\|_{d}$ and choose $\chi$ as in Lemma 4.5, with $\alpha^{ \pm}=u_{1, d}( \pm 1)$. Then we obtain by (4.29), (4.30),

$$
\Phi(d, S) \leq C\left(d^{\frac{1}{2}}+d p+p^{-1}\right) .
$$

Now since $\tilde{p} \leq K d^{-\frac{1}{2}}$, we have $d^{\frac{1}{2}} \leq K \tilde{p}^{-1}$. Substituting this in (4.32) gives (4.31).

Let us now put together the results of Theorems 4.1-4.3. The following theorem shows that the spaces $S(\Sigma)=\Pi_{p}(I)$ approximate boundary layers $u_{1, d}$ robustly at the rate $G(p)=C p^{-1} \sqrt{\ln p}$ in the energy norm (in the sense of Definition 3.2). Moreover, the best robust rate possible is $\mathrm{Cp}^{-1}$, so that the result established is optimal up to a factor $\sqrt{\ln p}$.

Theorem 4.4. Let $S(\Sigma)=\Pi_{p}(I)$. Then

$$
C p^{-1} \leq G(p)=\sup _{d \in(0,1]} \Phi(d, S) \leq C p^{-1} \sqrt{\ln p}
$$

where $C$ is a constant independent of $p$.

Proof. Let $d \in(0,1]$ be arbitrary. Suppose first that

$$
1 \leq \frac{\tilde{p}}{2 \sqrt{\ln p}} \leq \sqrt{\frac{3}{4 d}}, \quad \text { i.e., } \quad 1 \leq \tilde{p} \leq \sqrt{\frac{3 \ln p}{d}} .
$$

Then by Theorem 4.3, with $K=\sqrt{3 \ln p}$, we have in this range

$$
\Phi(d, S) \leq C K p^{-1} \leq C p^{-1} \sqrt{\ln p} .
$$


Next, for $\sqrt{3 /(4 d)} \leq \tilde{p} /(2 \sqrt{\ln p}) \leq 2 /(2 d \sqrt{\ln p})$ we may use Corollary 4.2 , by which

$$
\Phi(d, S) \leq C_{4} e^{-\tilde{p}^{2} d / 3}+C_{5}\left(\frac{e}{4}\right)^{\frac{2}{d}} .
$$

Since $\tilde{p} /(2 \sqrt{\ln p}) \geq \sqrt{3 /(4 d)}$, we have $\tilde{p}^{2} d / 3 \geq \ln p$, so that $\exp \left(-\tilde{p}^{2} d / 3\right) \leq 1 / p$. Also, since $2 / d \geq \tilde{p}$, it follows that $(e / 4)^{2 / d} \leq(e / 4)^{\tilde{p}} \leq p^{-1}$. Hence,

$$
\Phi(d, S) \leq C p^{-1}
$$

in this range. Finally, it is easy to see that the estimate for $\Phi(d, S)$ for the range $\tilde{p}>2 / d$, given by Theorem 4.1 , also satisfies (4.33).

To establish the lower bound, we note that by the triangle inequality, for any $\chi \in \Phi_{p}(I)$ with $\chi( \pm 1)=u( \pm 1)$

$$
\begin{aligned}
\Phi(d, S) & \geq\|\chi\|_{d}-\|u\|_{d} \\
& \geq\|\chi\|_{0}-\|u\|_{d} .
\end{aligned}
$$

If $d \rightarrow 0$, then $\|u\|_{d} \rightarrow 0$. But, by Lemma $4.5,\|\chi\|_{0} \geq C p^{-1}$, giving the result.

Suppose the $p$ version is used with a fixed mesh for problems (3.1). Then the rate $F(N, k)$ in $(3.11)$ satisfies

$$
F(N, k) \leq C p^{-(k-1)},
$$

so that the first infimum on the right side of (3.10) will certainly be less than $C p^{-1}$, uniformly in $d$ whenever $k \geq 2$. Using Theorem 4.4 on the whole interval $[-1,1]$, we can bound the infima involving boundary layers uniformly by $C p^{-1} \sqrt{\ln p}$ (the fact that we have more than one interval can only enhance this rate). Hence, the $p$ version is robust with uniform order $C p^{-1} \sqrt{\ln p}$. Moreover, this robust rate is optimal up to $\sqrt{\ln p}$, since for $d \rightarrow 0$, the approximation of the boundary layer terms in the end intervals cannot be better than $C p^{-1}$ by Theorem 4.4. We therefore have the following result.

Theorem 4.5. The $p$ version with fixed mesh for problems $(3.1), 0<d \leq 1$, is robust with uniform order $g(p)$ satisfying

$$
C p^{-1} \leq g(p) \leq C p^{-1} \sqrt{\ln p}
$$

with respect to solution sets $H_{d, M}^{B}\left(\right.$ or $\left.H_{d, \Pi_{n}}^{B}\right)$ and error measure the energy norm.

Remark 4.4. In terms of the number of degrees of freedom $N$, we see that $g(N) \approx$ $N^{-1} \sqrt{\ln N}$. This is essentially twice the best uniform rate of $N^{-1 / 2}$ that can be attained using the $h$ version with a quasiuniform mesh [13]. Hence, the "doubling" phenomenon for the rate of convergence for the $p$ version, which is well known for the case that $(x+1)^{\alpha}$ type singularities are present at $x=-1$ (see, e.g., [3]), also occurs when the solution contains boundary layer components of the type $\exp (-(x+1) / d)$ at $x=-1$.

\section{Approximation Results FOR AN $h p$ VERsion}

In the previous section, we showed that the $p$ version over a single element yields a super-exponential rate of convergence for $\tilde{p}>e / 2 d$. Also, the error decreases at the (exponential) rate $\exp \left(-\tilde{p}^{2} d / 3\right)$ in the preasymptotic range $\sqrt{3 /(4 d)} \leq \tilde{p} \leq 2 / d$ for small $d$. Unfortunately, in practice both these ranges may be difficult to achieve if $d$ is small and $p$ is restricted ( $p \leq 8$ is typical in programs such as MSC/PROBE 
and STRESSCHECK), so that all that may be observed is the uniform rate of $\mathcal{O}\left(p^{-1} \sqrt{\ln p}\right)$ predicted by Theorems $4.3,4$.4. In this section, we show that if only one extra element of size $\mathcal{O}(p d)$ is inserted in the boundary layer, then robust exponential convergence is achieved uniformly for $0<d \leq 1$ as $p$ increases. Since the mesh is changed at each step when $p$ is increased, we call this an $h p$ version FEM (more appropriately, it is an $r p$ version FEM). Naturally, if the polynomial degree $p$ is sufficiently large, we have to allow a transition to the single-element mesh analyzed in Theorem 4.1.

A more general question that could be considered is, given $N$ degrees of freedom $\left(N\right.$ as in (3.6)), for what mesh-degree combination $\Sigma$ (i.e., choice of $S_{D}$ ) is the error minimized? We do not consider this theoretical question here, since the simple two-element mesh below already gives exponential convergence, uniformly in $d$. This mesh is easier to implement than a general $h p$ version, and moreover, in computational experiments performed using meshes with more elements, we were unable to achieve better convergence rates (see $\S 6$ and [20]). Note that the meshdegree combination we propose is similar to the optimal mesh-degree combination obtained for a related problem by Scherer in [14] (see Remark 5.2).

The following theorem is our main result in this section.

Theorem 5.1. Let $I=(-1,1)$ and $u(x)=u_{1, d}=\exp (-(x+1) / d)$. Let further $\Sigma=(\Delta, \vec{p})$ be such that for $p \geq 1$

$$
\begin{aligned}
& \vec{p}=\{p, 1\}, \quad \Delta=\{-1,-1+\kappa \tilde{p} d, 1\} \quad \text { if } \kappa \tilde{p} d<2, \\
& \vec{p}=\{p\}, \quad \Delta=\{-1,1\} \quad \text { if } \kappa \tilde{p} d \geq 2,
\end{aligned}
$$

where $0<\kappa_{0} \leq \kappa<4 / e$ is a constant independent of $p$ and $d$. Then there exists $u_{p} \in S(\Sigma)$ such that $u_{p}( \pm 1)=u( \pm 1)$ and

$$
\left\|u-u_{p}\right\|_{d} \leq d^{1 / 2} C_{6} \alpha^{\tilde{p}}, \quad\left\|u-u_{p}\right\|_{0} \leq d^{1 / 2} C_{7} \alpha^{\tilde{p}}, \quad\left\|u^{\prime}-u_{p}^{\prime}\right\|_{0} \leq d^{-1 / 2} C_{8} \alpha^{\tilde{p}} .
$$

Here the constants are independent of $p$ and $d$ but depend on $\kappa_{0}$ and

$$
\alpha:=\left\{\begin{array}{cl}
e /(2 \tilde{p} d) & \text { if } \kappa \tilde{p} d \geq 2, \\
\max \left\{\kappa e / 4, e^{-(\kappa-\epsilon)}\right\} & \text { if } \kappa \tilde{p} d<2
\end{array}\right\}<1,
$$

with $\epsilon>\ln p /(2 p)$ arbitrary.

Proof. If $\kappa \tilde{p} d \geq 2$, we have that $r=e /(2 \tilde{p} d)<1$, owing to our assumption that $\kappa<$ $4 / e$. Therefore, Theorem 4.1 is applicable, and a $p$-increase in the single-element mesh $\Delta=\{-1,1\}$ yields exponential convergence with the number $r$ decreasing with $p$.

Consider now the case $\kappa \tilde{p} d<2$, i.e., the two-element mesh $\Delta=\{-1,-1+$ $\kappa \tilde{p} d, 1\}$. We assume first that $\tilde{p} \geq 2 / \kappa_{0}$ and construct the function $u_{p}(x) \in S(\Sigma)$ elementwise. Denote $I_{1}=(-1, a)$, where $a=-1+\kappa \tilde{p} d, \kappa_{0} \leq \kappa<4 / e$, and let $s_{1} \in \Pi_{p}\left(I_{1}\right)$. Transforming $I_{1}$ to $I=(-1,1)$, we see that for $t=0,1$,

$$
\int_{-1}^{a}\left(\frac{d^{t}}{d x^{t}}\left(u-s_{1}\right)\right)^{2} d x=\left(\frac{2}{\kappa \tilde{p} d}\right)^{2 t-1} \int_{-1}^{1}\left(\frac{d^{t}}{d y^{t}}\left(\tilde{u}-\tilde{s}_{1}\right)\right)^{2} d y .
$$

Here, $\tilde{f}(y)$ denotes the image on $I$ of any function $f(x)$ defined on $I_{1}$. Consequently, we obtain that $\tilde{u}(y)=\exp (-(y+1) \kappa \tilde{p} / 2)=u_{1, \tilde{d}}(y)$, where $\tilde{d}=2 / \kappa \tilde{p}$. 
Since $\kappa<4 / e$, we have $r:=e /(2 \tilde{p} \tilde{d})=\kappa e / 4<1$. Now Theorem 4.1 and Corollary 4.1 apply uniformly to functions $u \equiv u_{1, d}$ for all $d \in(0,1]$. Since $\tilde{p} \geq 2 / \kappa_{0} \geq$ $2 / \kappa$ we have that $\tilde{d}<1$, and hence Theorem 4.1 and Corollary 4.1 will apply when $d$ is chosen to be $\tilde{d}$. Then, since $r<1$, we obtain a polynomial $s_{p} \in \Pi_{p}\left(I_{1}\right)$ satisfying

$$
s_{p}(-1)=u(-1), \quad s_{p}(a)=u(a),
$$

and

$$
\begin{gathered}
\left\|\frac{d^{t}}{d x^{t}}\left(u-s_{p}\right)\right\|_{0, I_{1}}^{2} \leq C_{t}^{2}\left(\frac{2}{\kappa \tilde{p} d}\right)^{2 t-1} \tilde{d}^{1-2 t} \frac{r^{2 \tilde{p}}}{\left(1-r^{2}\right)}, \quad t=0,1, \\
\left(d^{2}\left\|u^{\prime}-s_{p}^{\prime}\right\|_{0, I_{1}}^{2}+\left\|u-s_{p}\right\|_{0, I_{1}}^{2}\right)^{1 / 2} \leq C_{2} d^{1 / 2} \frac{r^{\tilde{p}}}{\left(1-r^{2}\right)^{1 / 2}} .
\end{gathered}
$$

This gives the asserted bound on $I_{1}$ in the case $\tilde{p}>2 / \kappa_{0}$. Since this excludes only finitely many values of $p$, these estimates hold for all $p$ after possibly adjusting the constants $C_{t}, t=0,1,2$ in (5.4), (5.5).

As noted in Remark 4.1, the approximation $s_{p}(x)$ constructed via Lemma 4.1 is optimal in the $|\cdot|_{1}$ seminorm but not in the $\|\cdot\|_{d}$ norm. For fixed $d>0$, $s_{p}$ yields the optimal-order error as $p \rightarrow \infty$, but is suboptimal as $d \rightarrow 0$, owing to the enforcement of the interpolation condition (5.3). Therefore, we modify $s_{p}$ as follows: let $u_{p}=s_{p}-s_{1}+\tilde{s}_{1}$, where $s_{1}$ is the linear interpolant of $u_{1, d}(x)$ at $x=-1$ and $x=a$, and $\tilde{s}_{1}$ is a linear function such that $\tilde{s}_{1}(-1)=u(-1)$ and $\tilde{s}_{1}(a)=\max \left\{d^{1 / 2} u(a), u(1)\right\}$. Then

$$
\begin{aligned}
\left\|u-u_{p}\right\|_{d, I_{1}} & =\left\|u-\left(s_{p}-s_{1}+\tilde{s}_{1}\right)\right\|_{d, I_{1}} \\
& \leq\left\|u-s_{p}\right\|_{d, I_{1}}+\left\|s_{1}-\tilde{s}_{1}\right\|_{d, I_{1}} .
\end{aligned}
$$

The first term was estimated in (5.5), so we estimate the second term. We have

$$
\begin{aligned}
\int_{-1}^{a}\left(s_{1}-\tilde{s}_{1}\right)^{2} d x & \leq \max _{-1 \leq x \leq a}\left|\left(s_{1}-\tilde{s}_{1}\right)(x)\right|^{2}(1+a) \\
& \leq|(1-\sqrt{d}) u(a)|^{2}(1+a) .
\end{aligned}
$$

Since $1+a=\kappa \tilde{p} d$ and $u(a)=\exp (-(a+1) / d)=\exp (-\kappa \tilde{p})$, we get

$$
\left\|s_{1}-\tilde{s}_{1}\right\|_{0, I_{1}}^{2} \leq e^{-2 \kappa \tilde{p}} \kappa \tilde{p} d .
$$

Also,

$$
\int_{-1}^{a}\left(s_{1}^{\prime}-\tilde{s}_{1}^{\prime}\right)^{2} d x \leq(1-\sqrt{d})^{2}|u(a)|^{2}(1+a)^{-1} \leq \frac{e^{-2 \kappa \tilde{p}}}{\kappa \tilde{p} d} .
$$

Hence,

$$
d\left\|s_{1}^{\prime}-\tilde{s}_{1}^{\prime}\right\|_{0, I_{1}} \leq \frac{d^{1 / 2}}{\sqrt{\kappa \tilde{p}}} e^{-\kappa \tilde{p}}
$$

and altogether

$$
\begin{aligned}
\left\|s_{1}-\tilde{s}_{1}\right\|_{d} & \leq d^{\frac{1}{2}}\left(\kappa \tilde{p}+\frac{1}{\kappa \tilde{p}}\right)^{\frac{1}{2}} e^{-\kappa \tilde{p}} \\
& \leq d^{\frac{1}{2}}\left(\kappa+\frac{1}{\kappa}\right)^{\frac{1}{2}} e^{-(\kappa-\epsilon) \tilde{p}}
\end{aligned}
$$


for any $\epsilon>\frac{\ln \tilde{p}}{2 \tilde{p}}\left(\epsilon=\frac{1}{2 e}\right.$ works for all $\left.p\right)$. Then, from (5.5)-(5.7),

$$
\left\|u-u_{p}\right\|_{d, I_{1}}^{2} \leq \tilde{C}_{0}^{2} d\left\{\left(\frac{\kappa e}{4}\right)^{2 \tilde{p}}+e^{-2(\kappa-\epsilon) \tilde{p}}\right\} .
$$

Next we consider $I_{2}$. Here we select $u_{p} \in \Pi_{1}\left(I_{2}\right)$ to be the linear interpolant between $\max \left\{d^{1 / 2} u(a), u(1)\right\}$ at $x=a$ and $u(1)$ at $x=1$. One verifies that

$$
u_{p}(x)=(u(1)-\max \{u(1), \sqrt{d} u(a)\}) \frac{(x-a)}{1-a}+\max \{u(1), \sqrt{d} u(a)\} .
$$

Now let $\frac{2}{\kappa d} \geq \tilde{p} \geq \frac{2}{\kappa d}-\frac{|\ln d|}{2 \kappa}$. Then, since $\frac{|\ln d|}{2 \kappa} \leq \frac{e^{-1}}{2 \kappa d}$, we have $\tilde{p} \geq \frac{\left(4-e^{-1}\right)}{2 \kappa d}$ in this range. Also,

$$
u(1) \geq \sqrt{d} u(a) \text { and } 1-a \leq \frac{|\ln d| d}{2} \leq \ln \left(\frac{2 \tilde{p} \kappa}{\left(4-e^{-1}\right)}\right) \frac{d}{2} .
$$

Hence,

$$
\begin{gathered}
\int_{a}^{1} u_{p}^{2} d x \leq u^{2}(1) \frac{|\ln d| d}{2} \leq \frac{d}{2} \ln \left(\frac{2 \tilde{p} \kappa}{\left(4-e^{-1}\right)}\right) e^{-2 \kappa \tilde{p}} \leq \tilde{C}_{1} d e^{-2(\kappa-\epsilon) \tilde{p}} \\
\int_{a}^{1}\left(u_{p}^{\prime}\right)^{2}=0 .
\end{gathered}
$$

Next, for $\tilde{p}<\frac{2}{\kappa d}-\frac{|\ln d|}{2 \kappa}$

$$
u(1)<\sqrt{d} u(a) \quad \text { and } \quad \frac{1}{1-a} \leq \frac{2}{d|\ln d|} .
$$

Hence,

$$
\begin{gathered}
\int_{a}^{1} u_{p}^{2} d x \leq(\sqrt{d} u(a))^{2}(1-a) \leq 2 d e^{-2 \kappa \tilde{p}} \\
\int_{a}^{1}\left(u_{p}^{\prime}\right)^{2} d x \leq \frac{2 d u^{2}(a)}{|\ln d| d} \leq \frac{2 e^{-2 \kappa \tilde{p}}}{|\ln d|}
\end{gathered}
$$

For $d \leq e^{-1}$, this gives

$$
d^{2} \int_{a}^{1}\left(u_{p}^{\prime}\right)^{2} d x \leq 2 d^{2} e^{-2 \kappa \tilde{p}} .
$$

For $e^{-1}<d \leq 1$, we have by (5.9) that

$$
\int_{a}^{1}\left(u_{p}^{\prime}\right)^{2} d x \leq 2\left(\frac{(u(1)-u(a))^{2}}{1-a}+\frac{(1-\sqrt{d})^{2} u^{2}(a)}{1-a}\right) .
$$

By the Mean Value Theorem, there exists $\xi \in[a, 1]$ such that $u(1)-u(a)=$ $u^{\prime}(\xi)(1-a)$, so that

$$
\begin{aligned}
d^{2} \int_{a}^{1}\left(u_{p}^{\prime}\right)^{2} d x & \leq 2 e^{-2\left(\frac{\xi+1}{d}\right)}(1-a)+4 d \frac{(1-\sqrt{d})^{2} u^{2}(a)}{|\ln d|} \\
& \leq \tilde{C}_{2} d e^{-2 \kappa \tilde{p}}
\end{aligned}
$$


uniformly as $d \rightarrow 1$, where $\tilde{C}_{2}$ may be explicitly evaluated. Hence, we conclude by (5.10)-(5.13) that

$$
\left\|u_{p}\right\|_{d, I_{2}}^{2} \leq \tilde{C}_{3} d e^{-2(\kappa-\epsilon) \tilde{p}} .
$$

Also, it is easy to verify that

$$
\|u\|_{0, I_{2}}^{2} \leq \frac{d}{2} e^{-2 \kappa \tilde{p}}, \quad\left\|u^{\prime}\right\|_{0, I_{2}}^{2} \leq \frac{2}{d} e^{-2 \kappa \tilde{p}},
$$

so that by (5.14), (5.15) and the triangle inequality,

$$
\left\|u-u_{p}\right\|_{d, I_{2}}^{2} \leq \tilde{C}_{4} d e^{-2(\kappa-\epsilon) \tilde{p}} .
$$

Then the first inequality in (5.1) follows from Theorem 4.1, (5.8), (5.16). The other two inequalities also follow from the estimates above.

Remark 5.1. The constant $\kappa$ in Theorem 5.1 could be selected such that $\kappa^{*} e=$ $4 e^{-\kappa^{*}}$, which yields $\kappa^{*} \approx 0.71$. This gives $\alpha \approx e^{-\kappa^{*}}$ in $(5.2)$ when two elements are being used. This value for $\kappa^{*}$ is, however, not optimal since it is obtained by optimizing some upper bounds. The optimal choice of $\kappa$ is numerically addressed in $\S 6$ ahead. Use of the above value of $\kappa^{*}$, however, simplifies the bounds above.

Remark 5.2. The choice of $\Sigma=(\Delta, \tilde{p})$ used in Theorem 5.1 is similar to that obtained by Scherer in [14]. He considered the best mesh-degree combination (for a fixed number of degrees of freedom $N$ ) that would minimize the $L^{\infty}$ error of best approximation (by discontinuous piecewise polynomials) of the function $e^{-x}$ on the interval $[0, \infty)$. He was able to solve this problem explicitly - the asymptotically optimal $\Sigma$ was given by $\Delta=\left\{0, q_{0}(p+1), \infty\right\}, \vec{p}=\{p, 1\}$, where $p=N-2$ and $q_{0}=0.89548641 \ldots$. For this $\Sigma$, Scherer showed that the asymptotic $L^{\infty}$ convergence rate was $e^{-q_{0} N}=e^{-q_{0}(p+2)}$, which (up to an algebraic factor in $N$ ) was, asymptotically, the best possible for any mesh-degree combination.

We can also deduce pointwise error bounds.

Corollary 5.1. Under the assumptions of Theorem 5.1 we have

$$
\left\|u-u_{p}\right\|_{L^{\infty}(I)} \leq C_{9} \alpha^{\tilde{p}}
$$

with $\alpha$ as in Theorem 5.1.

Proof. This follows from (5.1) and the interpolation inequality

$$
\|v\|_{L^{\infty}(I)} \leq 2\|v\|_{L_{2}(I)}^{1 / 2}\left\|v^{\prime}\right\|_{L_{2}(I)}^{1 / 2}
$$

Remark 5.3. The estimates in Theorem 5.1, Corollary 5.1 are obtained using polynomials of degree 1 in $I_{2}$. They evidently remain valid if $I_{2}$ is subdivided and/or the degree $p$ is greater than 1 in $I_{2}$.

Theorem 5.1 says that it is sufficient to use two intervals of the type described to resolve boundary layers with a robust exponential convergence rate. As discussed in $\S 2$, the solution will typically have other (smoother) components as well. For the approximation of these components, the mesh-degree combination of Theorem 5.1 will typically not be sufficient and will have to be enhanced (e.g., by subdivision or $p$-increase in element 2). This enhancement will ensure that the rate $F(N, k)$ in (3.11), which measures the approximation of these smoother components, is sufficiently rapid. For solutions in $H_{d, M}^{B}$, the robust rate of convergence $g(N)$ of the $h p$ version will then be given by (3.14), where $G(N)$ represents the exponential 
rate (5.1). As noted in Remark 3.1, the overall rate will be exponential only if the smooth components are also approximated exponentially. One such case occurs when $f$ is a polynomial, as noted in the theorem below. (Note that we have a boundary layer at each end-point now.)

Theorem 5.2. Consider the $h p$ version for problem (3.1), $0<d \leq 1$, with $\Sigma=$ $(\Delta, \vec{p})$ given by

$$
\begin{aligned}
& \vec{p}=\{p, p, p\}, \quad \Delta=\{-1,-1+\kappa \tilde{p} d, 1-\kappa \tilde{p} d, 1\} \quad \text { if } \kappa \tilde{p} d<1, \\
& \vec{p}=\{p, p\}, \quad \Delta=\{-1,0,1\} \quad \text { if } \kappa \tilde{p} d \geq 1,
\end{aligned}
$$

where $\kappa$ is as in Theorem 5.1. Let $\alpha<1$ be defined as in (5.2) (with the condition $\kappa \tilde{p} d<2$ replaced by $\kappa \tilde{p} d<1$ ). Then there exists a constant $C>0$ independent of $p$ and $d$ such that with respect to solution sets $H_{d, \Pi_{n}}^{B}$ and error measure the energy norm, this version is robust with uniform order $g(p)=C d^{1 / 2} \alpha^{\tilde{p}}$ for $p \geq n$.

Proof. The theorem follows easily by (3.10), Corollary 2.1 and Theorem 5.1.

\section{Numerical RESUlts}

In this section, we present the results of numerical computations for the model problem (1.2)-(1.3), where:

$$
f(x) \equiv 1, \quad \alpha^{+}=\alpha^{-}=0, \quad a=1 .
$$

The exact solution is then given by

$$
u_{d}(x)=1-\frac{\cosh (x / d)}{\cosh (1 / d)}
$$

so that

$$
\left\|u_{d}\right\|_{d}^{2}=B_{d}\left(u_{d}, u_{d}\right)=\left(1, u_{d}\right)=2-2 d \tanh (1 / d)=\mathcal{O}(1) .
$$

Note that since $f(x)$ is a polynomial of degree 0, Corollary 2.1 applies. Noting (6.3), we conclude that the relative error in the energy norm,

$$
E_{R}(d)=\left\|u_{d}-u_{d}^{S}\right\|_{d} /\left\|u_{d}\right\|_{d}
$$

should behave like $\Phi(d, S)$ given by (3.12). All graphs shown in this section will depict $E_{R}(d)$ versus the number of degrees of freedom in the finite element method. The value of $d$ (and, where applicable, of $\kappa$ ) will be stated with the figures. All computations were done in double precision on an SGI indigo2 workstation using MATLAB 4.1.

We first consider the $p$ version over a single element. Figure 2 shows $E_{R}(d)$ plotted versus the number of degrees of freedom $N=p-1$, for various values of $d$, in a semilog scale. By Corollary 4.1 , for $\tilde{p}>e / 2 d$, the error will be in the asymptotic (superexponential) range. This is only reached, however, when $p \approx 13$ for $d=0.1$, $p \approx 136$ for $d=0.01, p \approx 1359$ for $d=0.001$ and $p \approx 13,591$ for $d=0.0001$. We see that, except for the first value, none of the rest can be considered as within a practical range of $p$. For $d=0.1$, however, the graph in the semilog scale of Figure 2 is close to a straight line for $p$ in this range, showing agreement with the theory.

Turning to the case $d=0.01$, we note that Corollary 4.2 predicts for $d$ small and $\sqrt{3 /(4 d)} \leq \tilde{p} \leq 2 / d$, i.e., $5 \leq p \leq 200$, that $\log \left(E_{R}\right)$ should behave like $-\gamma \tilde{p}^{2} d$, where $\gamma>0$ is independent of $d$ (the value of $\gamma$ in Corollary 4.2 is $1 / 3$, but this may 


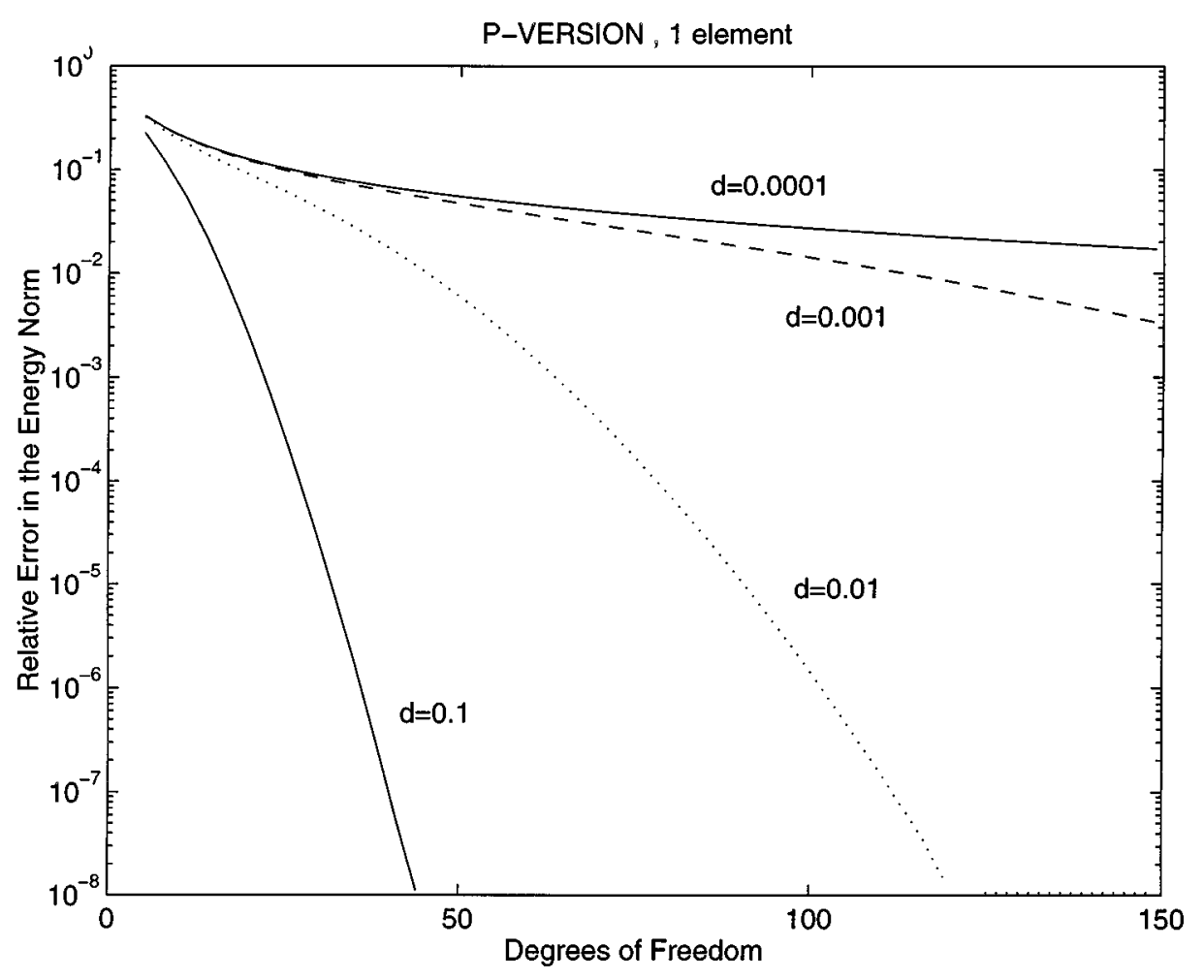

FiguRE 2. The $p$ version with one element

not be optimal). Hence, we should observe a parabolic curve for $d=0.01$ when $p$ is large enough. Again, the graph in Figure 2 is consistent with this bound.

As $d$ becomes even smaller, the error in Figure 2 is seen to deteriorate further. For $1 \leq \tilde{p} \leq K d^{-\frac{1}{2}}$, Theorem 4.3 predicts a convergence rate of only $C K p^{-1}$. This is precisely what is observed in Figure $6\left(d=10^{-3}\right)$ and Figure $7\left(d=10^{-6}\right)$ ahead. The graphs are now in a $\log -\log$ scale, and we observe straight lines with slope -1 . The "doubling" over the rate of convergence with the uniform $h$ version is also clearly apparent from these figures.

Let us now consider the $h p$ version, i.e., the $p$ version on a variable mesh. Since our model solution (6.1) has a boundary layer at each endpoint of the domain, the minimum number of elements as in Theorem 5.2 will now be 3 , with $\Sigma$ given by (5.18). (Since $f$ is a polynomial of degree 0 , we can actually take the minimal degree vector to be $\vec{p}=\{p, 1, p\}$.) From Theorem 5.2, we have the error estimate

$$
E_{R} \leq C(\kappa) d^{1 / 2} \alpha^{N / 2}, \quad N=\operatorname{dim}\left(S_{0}(\Sigma)\right)=2 p+1,
$$

with $\alpha$ given by (5.2). The experiments in Figure 3, obtained with $\kappa=\kappa^{*}=0.71$, clearly show the uniform exponential convergence as well as the factor $d^{1 / 2}$, since $\log \left(E_{R}(d)\right)$ plotted against $N$ is a straight line, which translates downwards as $d$ decreases. By Remark 5.1, for $\kappa=0.71$, we have $\alpha \approx e^{-0.71}$ for $p$ large - this is the same value that emerges by measuring the slopes in Figure 3. Note that the striking accuracy obtained for small $d$ is not possible with a comparable number of 


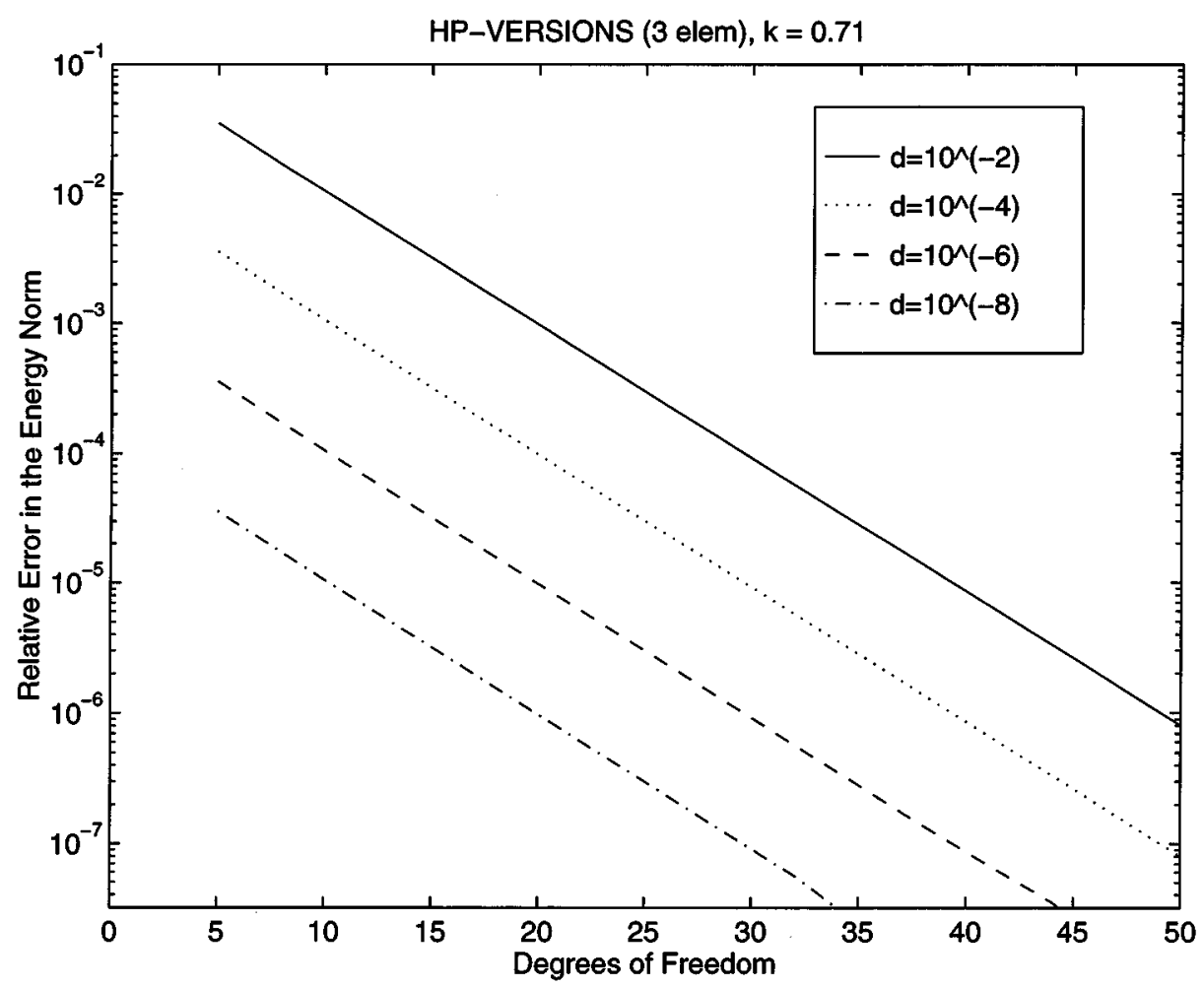

FiguRE 3. The $h p$ version for three elements with $\kappa=0.71$

degrees of freedom and methods based on a single element (see, e.g., the results in $[7,5])$.

In Figure 4, we investigate the convergence of the three-element $h p$ version for different values of $\kappa$, when $d=10^{-6}$ (other values of $d$ show similar results). We observe that $\kappa=\kappa^{*}=0.71$ is not quite optimal, since $\kappa=1$ gives better results. Careful examination shows that the graph for $\kappa=1$ consists of two linear pieces with different slopes. This is due to the fact that initially, the error in the central interval is dominant, so that the value of $\alpha$ in (5.2), (6.3) is close to $e^{-\kappa}$. As $p$ increases, the size of this interval decreases and the error in the other two intervals eventually dominates, with $\alpha$ behaving like $\kappa e / 4$. (Recall that we obtained $\kappa^{*}$ by setting $e^{-\kappa}$ equal to $\kappa e / 4$, so that only one straight line is observed in this case.)

Finally, in Figures $5-7$, we show a performance comparison between the various methods for $d=10^{-2}, 10^{-3}$ and $10^{-6}$, respectively (smaller values of $d$ up to $10^{-8}$ were tested, for which the behavior was similar to $d=10^{-6}$ ). In these figures, we have shown the results with four methods: (a) the $p$ version with one element, (b) the $h$ version with $p=1$, (c) the $h p$ version with 3 elements taking $\vec{p}=(p, p, p)$ and $\kappa=1$ and $(\mathrm{d})$ the $h$ version (taking $p=1)$ with the exponential mesh $\Delta=\left\{-1, x_{1}, \ldots, x_{m-1}, 1\right\}$, where, for $m$ even,

$$
x_{\frac{m}{2} \pm i}=\mp d \tilde{p} \ln \left(1-c \frac{2 i}{m}\right), \quad i=0, \ldots, \frac{m}{2},
$$




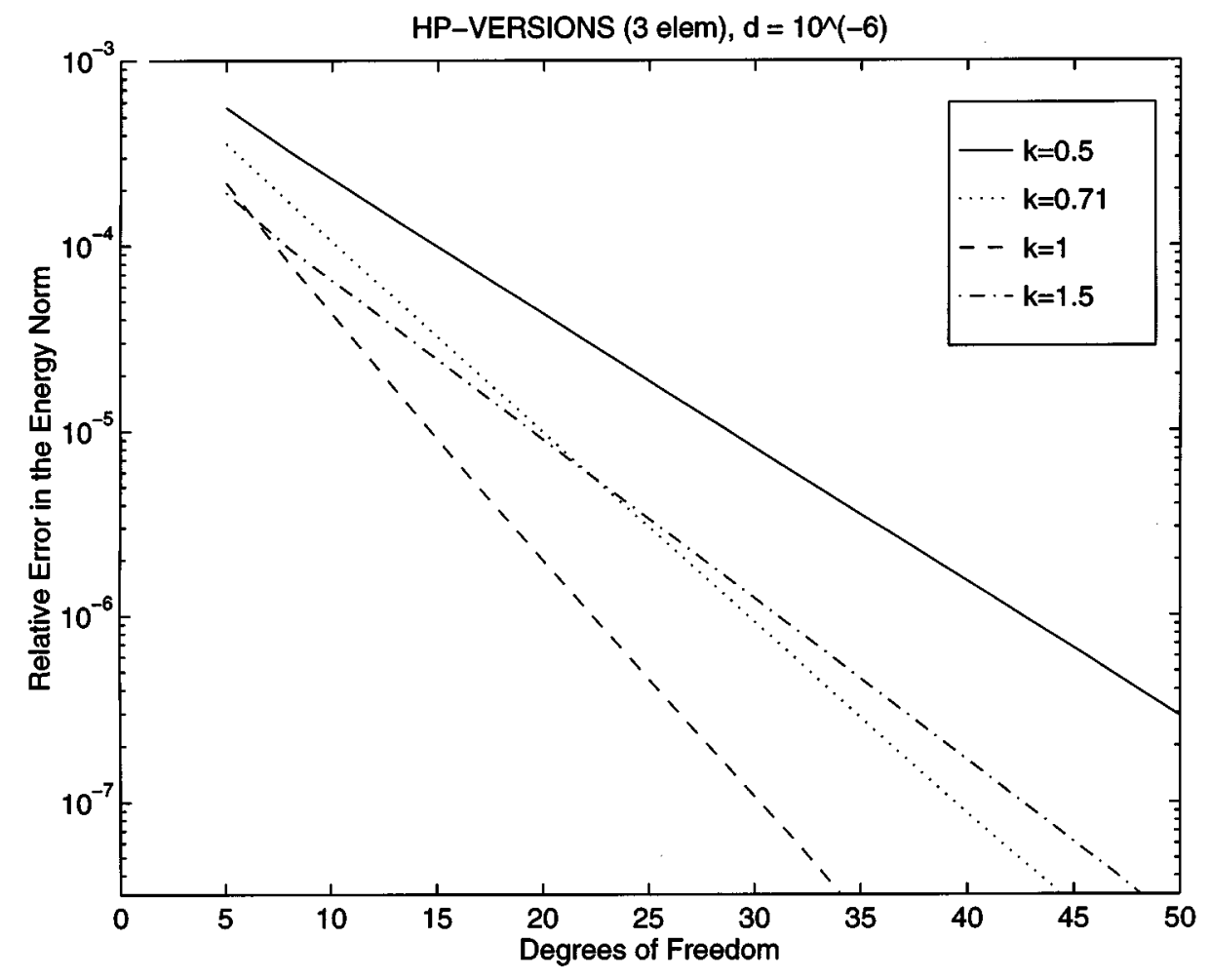

FiguRE 4. The dependence on parameter $\kappa$

with $c=1-\exp (-1 /(d \tilde{p}))$. The mesh $(6.5)$ is derived in $[16,20]$. We observe the following.

(i) The uniform $h$ version converges with order $\mathcal{O}\left(N^{-1 / 2}\right)$, the $p$ version on a single element with order $\mathcal{O}\left(N^{-1}\right)$, and the $h$ version with exponential mesh at the optimal algebraic rate of $\mathcal{O}\left(N^{-1}\right)$.

(ii) Both the $h$ version with exponential mesh and the $h p$ version have an error which behaves like $\mathcal{O}\left(d^{1 / 2}\right)$ in dependence on $d$. The other two versions do not display this translation as $d \rightarrow 0$.

(iii) For $d=10^{-2}$, the $p$ version rapidly reaches a superexponential rate, and eventually becomes the method with the fastest convergence. Asymptotically, i.e., for $\kappa \tilde{p} d>2$ and fixed $d$, the $p$ version with a single element will always have the best convergence rate according to Theorems 4.1 and 5.1. Accordingly, Theorem 5.1 indicates that at about $\kappa \tilde{p} d=2$ one must switch from the $h p$ version to a single-element $p$ version. For $d=10^{-2}$, this is apparent in Figure 5, where the one-element $p$ version becomes superior at some point. However, as is clearly visible in Figures 6 and 7, this point may occur so late that the only feasible method (in the practical range of $p$ ) is the three-element $h p$ version. 


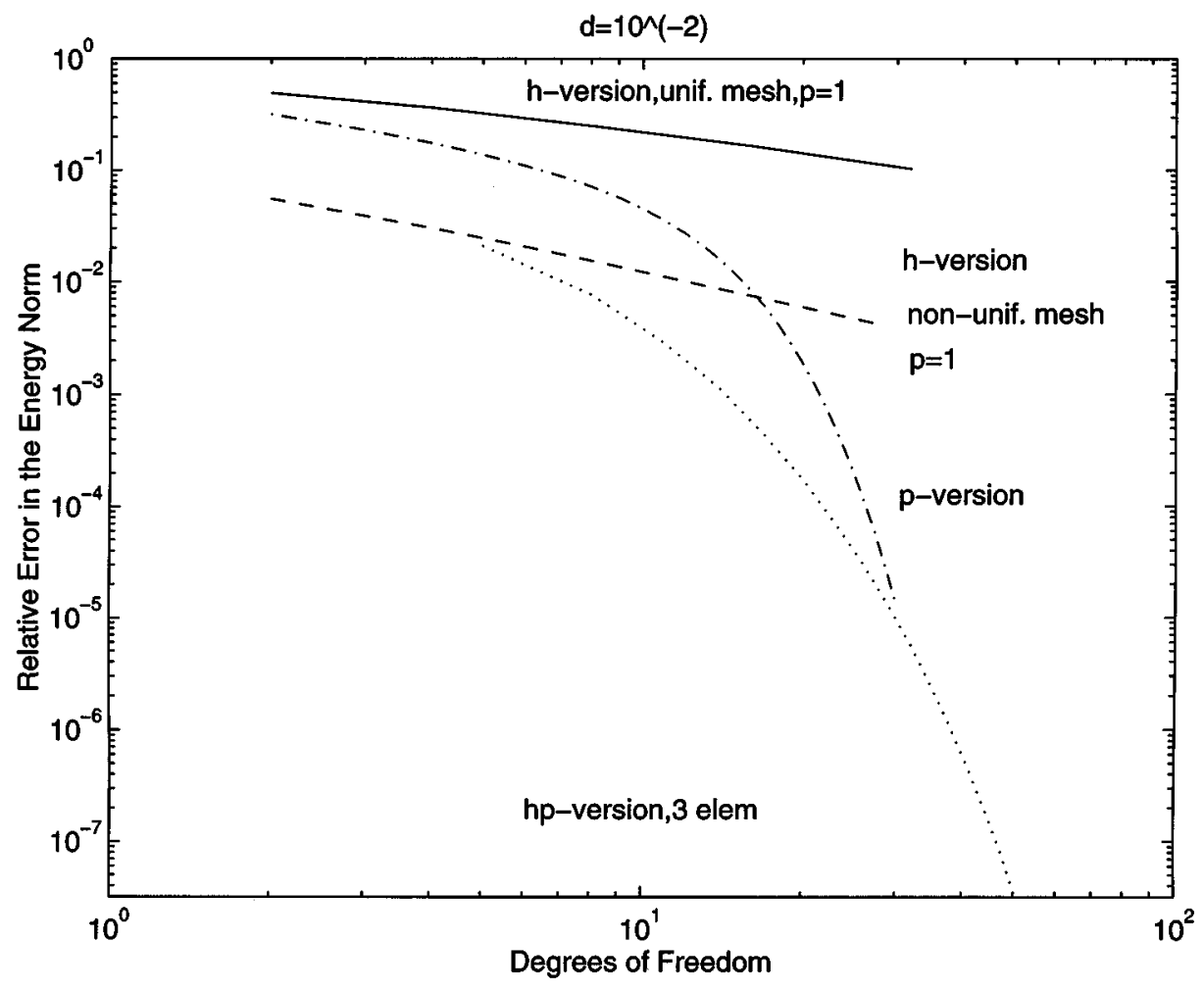

Figure 5. Comparison of various methods, $d=10^{-2}$

\section{APPENDIX}

We prove Theorem 2.1. For $M \in \mathbb{N}$, we define

$$
\omega_{d}^{M}(x)=\sum_{k=0}^{M} d^{2 k} a^{-2 k-2} f^{(2 k)}(x) .
$$

Then we see, using (1.2), that $R_{d}^{M}=u_{d}-\omega_{d}^{M}$ satisfies

$$
L_{d} R_{d}^{M}=f-L_{d} \omega_{d}^{M}=(d / a)^{2 M+2} f^{(2 M+2)}(x)=: g(x) .
$$

For $M$ large, we see that $\omega_{d}^{M}$ will satisfy (1.2) up to the correction $g(x)$. However, in general, the boundary conditions (1.3) will not be satisfied. We therefore introduce appropriate boundary layer terms to enforce (1.3). For this purpose, we define $u_{k}^{B L}(x)$ to be the unique solution of

$$
L_{d} u_{k}^{B L}(x)=0 \quad \text { on } I
$$

$$
u_{k}^{B L}( \pm 1)=C_{k}^{ \pm}:=\delta_{0, k} \alpha^{ \pm}-a^{-2 k-2} f^{(2 k)}( \pm 1) .
$$

Then with $u_{a, d}$ and $\bar{u}_{a, d}$ as defined in (2.4), we may verify that

$$
u_{k}^{B L}(x)=A_{k} u_{a, d}(x)+B_{K} \bar{u}_{a, d}(x),
$$




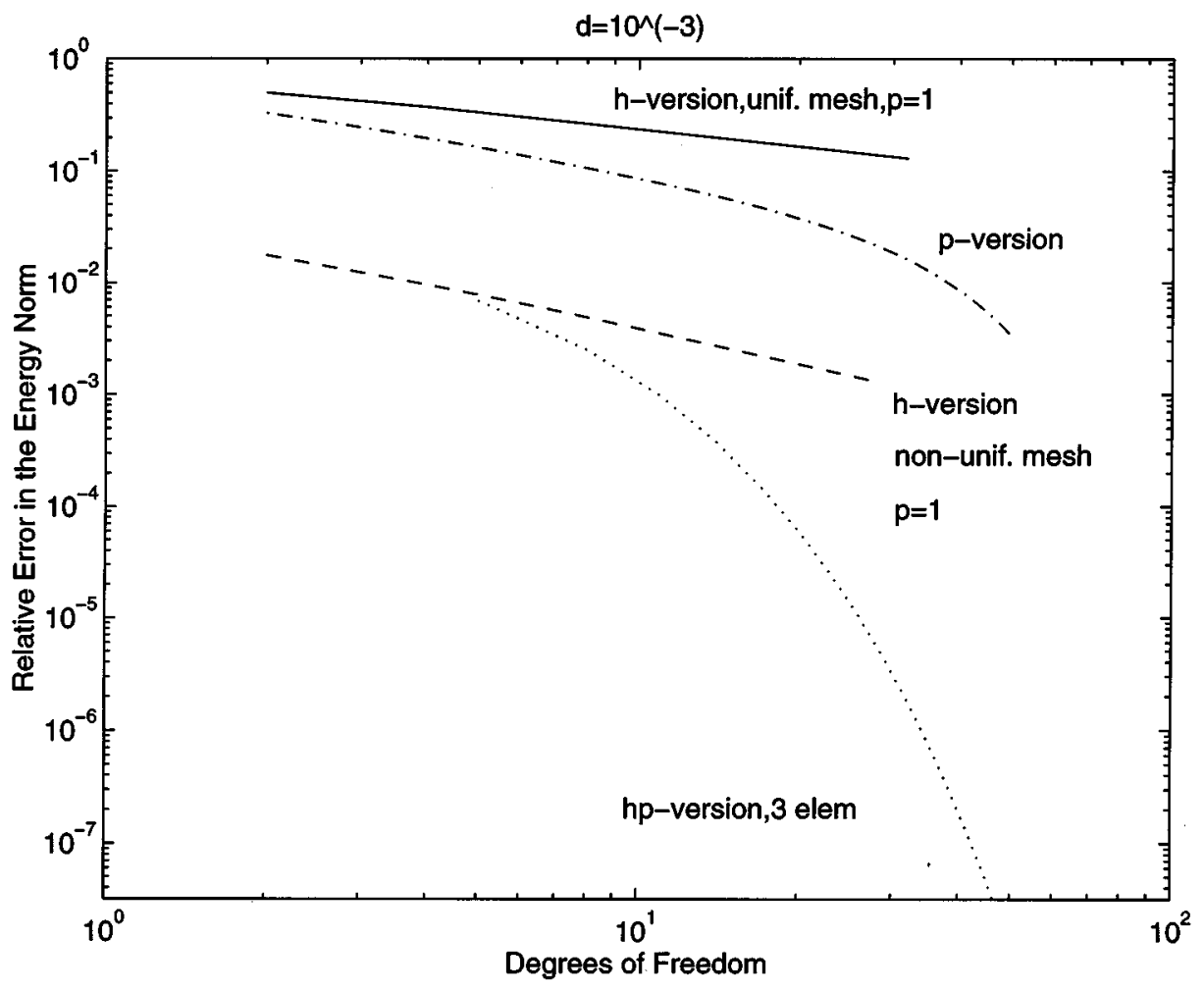

Figure 6. Comparison of various methods, $d=10^{-3}$

where

$$
A_{k}=\frac{C_{k}^{-}-C_{k}^{+} e^{-2 a / d}}{1-e^{-4 a / d}}, \quad B_{k}=\frac{C_{k}^{+}-C_{k}^{-} e^{-2 a / d}}{1-e^{-4 a / d}} .
$$

Then we write

$$
U_{M}^{B L}(x)=\sum_{k=0}^{M} d^{2 k} u_{k}^{B L}(x)=A_{d}^{M} u_{a, d}(x)+B_{d}^{M} \bar{u}_{a, d}(x),
$$

where $A_{d}^{M}=\sum_{k=0}^{M} d^{2 k} A_{k}$ and $B_{d}^{M}=\sum_{k=0}^{M} d^{2 k} B_{k}$. We see that

$$
\left|A_{d}^{M}\right| \leq \sum_{k=0}^{M} d^{2 k}\left|A_{k}\right| \leq\left(1-e^{-4 a}\right)^{-1} \sum_{k=0}^{M} d^{2 k}\left(\left|C_{k}^{-}\right|+\left|C_{k}^{+}\right|\right),
$$

with a similar bound holding for $\left|B_{d}^{M}\right|$. Equation (2.7) follows from (6.12) and (6.9).

We now define

$$
r_{d}^{M}=u_{d}-\omega_{d}^{M}-A_{d}^{M} u_{a, d}-B_{d}^{M} \bar{u}_{a, d}=R_{d}^{M}-U_{M}^{B L} .
$$

Then $r_{d}^{M} \in H_{0}^{1}(I)$ and $r_{d}^{M}$ satisfies (6.7). Hence,

$$
\left\|r_{d}^{M}\right\|_{E}^{2}:=B_{d}\left(r_{d}^{M}, r_{d}^{M}\right)=\left(g, r_{d}^{M}\right) \leq\|g\|_{0}\left\|r_{d}^{M}\right\|_{E} .
$$




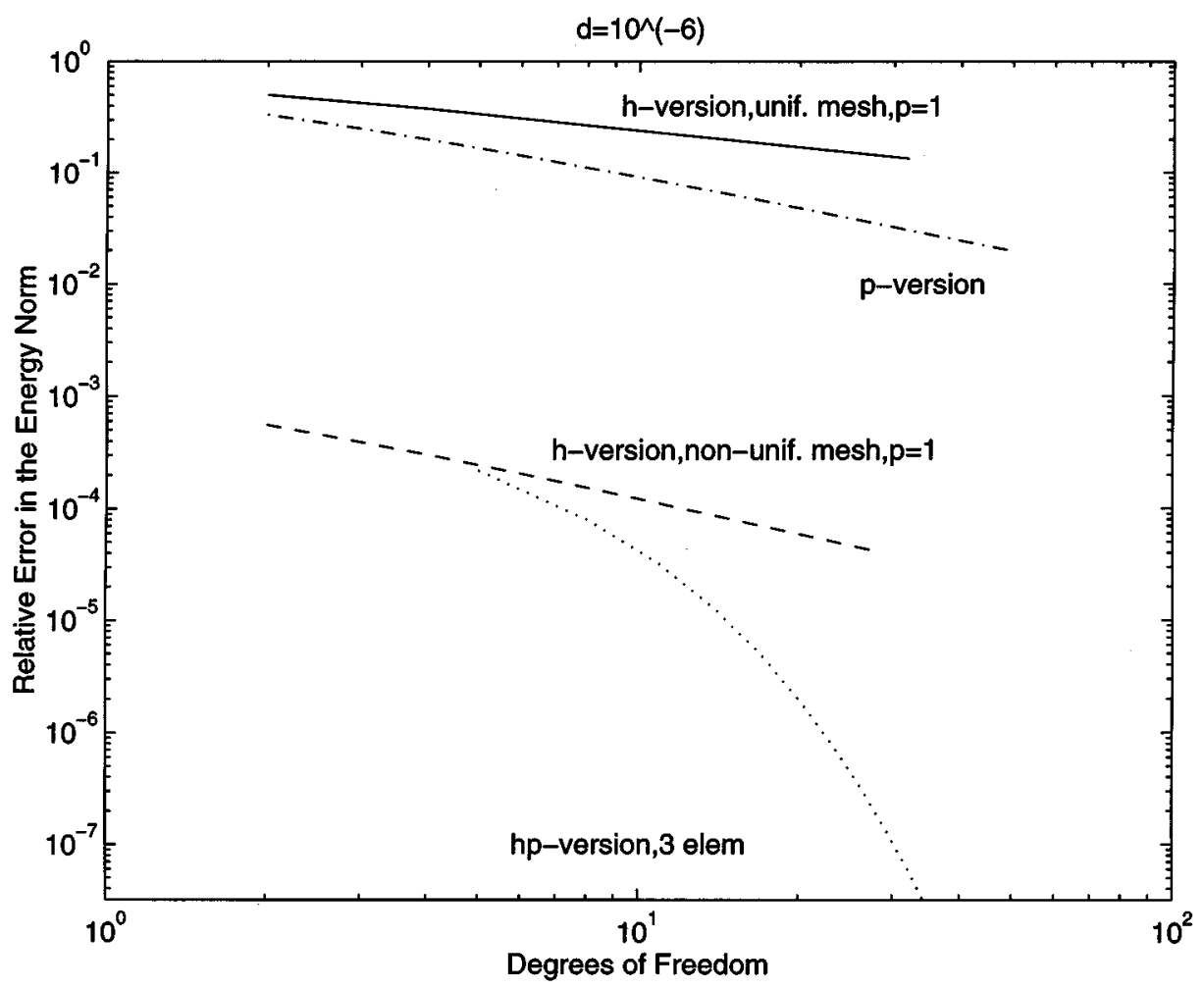

Figure 7. Comparison of various methods, $d=10^{-6}$

From this we deduce (using (6.7)) that

$$
\left\|r_{d}^{M}\right\|_{0} \leq a^{-1}(d / a)^{2 M+2}\left\|f^{(2 M+2)}\right\|_{0}, \quad\left|r_{d}^{M}\right|_{1} \leq a^{-1}(d / a)^{2 M+1}\left\|f^{(2 M+2)}\right\|_{0} .
$$

Since $r_{d}^{M}$ satisfies (6.7), we may differentiate (6.7) successively to obtain, using $(6.13)$,

$$
\left|r_{d}^{M}\right|_{\ell} \leq a^{-1}(d / a)^{2 M+2-\ell}\left\|f^{(2 M+2)}\right\|_{0}+a^{-2} \sum_{k=0}^{\ell-2}(d / a)^{2 M+2-\ell+k}\left\|f^{(2 M+2+k)}\right\|_{0},
$$

where $\ell=0,1, \ldots, 2 M$. Moreover, from (6.6), we see that for $\ell=0,1, \ldots, 2 M$

$$
\left|\omega_{d}^{M}\right|_{\ell} \leq a^{-2} \sum_{k=0}^{M}(d / a)^{2 k}\left\|f^{(2 k+\ell)}\right\|_{0} .
$$

Define $u_{d}^{M}=\omega_{d}^{M}+r_{d}^{M}$. Then (2.5) holds. Also, using (6.14)-(6.15), we may establish (2.6).

Remark 6.1. Suppose $f \in \Pi_{2 M+1}(I)$. Then in (6.7), we have $g(x) \equiv 0$, so that, since $r_{d}^{M} \in H_{0}^{1}(I)$ satisfies (6.7), we must have $r_{d}^{M}=0$. Hence, $u_{d}^{M}=\omega_{d}^{M}$, and it is seen by (6.6) that $u_{d}^{M} \in \Pi_{2 M+1}(I)$. 


\section{ACKNOWLEDGEMENTS}

The authors would like to thank Christos Xenophontos for the computations performed in Section 6, and Professor F. W. J. Olver for helpful discussions regarding the reference [9].

\section{REFERENCES}

1. D. N. Arnold and R. S. Falk. Asymptotic analysis of the boundary layer for the ReissnerMindlin plate model. SIAM J. Math. Anal. 27: 486-514, 1996.

2. I. Babuška and M. Suri. On locking and robustness in the finite element method. SIAM J. Numer. Anal., 29:1261-1293, 1992. MR 94c:65128

3. I. Babuška and B. A. Szabo. Lecture notes on finite element analysis, (to appear).

4. I. A. Blatov and V. V. Strygin. On estimates best possible in order in the Galerkin finite element method for singularly perturbed boundary value problems. Russian Acad. Sci. Dokl. Math., 47:93-96, 1993.

5. C. Canuto. Spectral methods and a maximum principle. Math. Comp., 51:615-629, 1988. MR 89d:65099

6. E. C. Gartland. Uniform high-order difference schemes for a singularly perturbed two-point boundary value problem. Math. Comp., 48:551-564, 1987. MR 89a:65116

7. W. B. Liu and J. Shen. A new efficient spectral Galerkin method for singular perturbation problems, Preprint, Department of Mathematics, Penn State University, State College Pa (1994).

8. W. B. Liu and T. Tang. Boundary layer resolving methods for singularly perturbed problems, submitted to I.M.A. J. Numer. Anal.

9. F. W. J. Olver. Error bounds for the Liouville-Green (or WKB) approximation. Proc. Cambridge Philos. Soc., 57:790-810, 1961. MR 24:A313

10. I. S. Gradshteyn and I. M. Ryzhik. Table of Series, Integrals and Products, enlarged edition. Wiley, New York, 1980. MR 81g:33001

11. H. Hakula, Y. Leino, and J. Pitkäranta. Scale resolution, layers and high-order numerical modeling of shells, to appear in Comp. Meth. Appl. Mech. Eng., 1996.

12. H. Kraus. Thin elastic shells: an introduction to the theoretical foundations and the analysis of their static and dynamic behavior. New York, Wiley 1967.

13. A. H. Schatz and L. B. Wahlbin. On the finite element method for singularly perturbed reaction-diffusion problems in two and one dimensions. Math. Comp., 40:47-89, 1983. MR 84c: 65137

14. K. Scherer. On optimal global error bounds obtained by scaled local error estimates. Numer. Math, 36:151-176, 1981. MR 82e:65053

15. C. Schwab and M. Suri. Locking and boundary layer effects in the finite element approximation of the Reissner-Mindlin plate model. Proc. Symp. Appl. Math., 48:367-371, 1994. MR 95m:65195

16. C. Schwab, M. Suri, and C. Xenophontos. The $h p$ finite element method for problems in mechanics with boundary layers (to appear).

17. C. Schwab and S. Wright. Boundary layers in hierarchical beam and plate models, report. Journal of Elasticity 38 (1995), 1-40. MR 96d:73044

18. G.I. Shishkin. Grid approximation of singularly perturbed parabolic equations with internal layers. Soviet J. Numer. Anal. Math. Modelling, 3:393-407, 1988. MR 89k:65109

19. R. Vulanović, D. Herceg, and N. Petrović. On the extrapolation for a singularly perturbed boundary value problem. Computing, 36:69-79, 1986. MR 88a:65008

20. C. A. Xenophontos. The $h p$ version of the finite element method for singularly perturbed problems in unsmooth domains. Ph.D. Dissertation, UMBC, 1996.

Seminar for Applied Mathematics, ETH Zürich, RÄmistrasse 101, CH-8092, Zürich, SWITZERLAND

E-mail address: schwab@sam.math.ethz.ch

Department of Mathematics and Statistics, University of Maryland Baltimore County, 5401 Wilkens Avenue, Baltimore, Maryland 21228

E-mail address: suri@math.umbc.edu 